\title{
Mobility of an axisymmetric particle near an elastic interface
}

\author{
Abdallah Daddi-Moussa-Ider ${ }^{1, \dagger}$, , Maciej Lisicki ${ }^{2,3} \ddagger$ and Stephan Gekle ${ }^{1}$ \\ ${ }^{1}$ Biofluid Simulation and Modeling, Fachbereich Physik, Universität Bayreuth, Universitätsstraße 30, \\ Bayreuth 95440, Germany \\ ${ }^{2}$ Department of Applied Mathematics and Theoretical Physics, University of Cambridge, Wilberforce Rd, \\ Cambridge CB3 OWA, UK \\ ${ }^{3}$ Institute of Theoretical Physics, Faculty of Physics, University of Warsaw, Pasteura 5, \\ 02-093 Warsaw, Poland
}

(Received 29 July 2016; revised 12 October 2016; accepted 2 November 2016)

Using a fully analytical theory, we compute the leading-order corrections to the translational, rotational and translation-rotation coupling mobilities of an arbitrary axisymmetric particle immersed in a Newtonian fluid moving near an elastic cell membrane that exhibits resistance towards stretching and bending. The frequency-dependent mobility corrections are expressed as general relations involving separately the particle's shape-dependent bulk mobility and the shape-independent parameters such as the membrane-particle distance, the particle orientation and the characteristic frequencies associated with shearing and bending of the membrane. This makes the equations applicable to an arbitrary-shaped axisymmetric particle provided that its bulk mobilities are known, either analytically or numerically. For a spheroidal particle, these general relations reduce to simple expressions in terms of the particle's eccentricity. We find that the corrections to the translation-rotation coupling mobility are primarily determined by bending, whereas shearing manifests itself in a more pronounced way in the rotational mobility. We demonstrate the validity of the analytical approximations by a detailed comparison with boundary integral simulations of a truly extended spheroidal particle. They are found to be in a good agreement over the whole range of applied frequencies.

Key words: biological fluid dynamics, blood flow, capsule/cell dynamics, membranes

\section{Introduction}

Hydrodynamic interactions between nanoparticles and cell membranes play an important role in many medical and biological applications. Prime examples are drug delivery and targeting via nanocarriers which release the active agent in disease sites such as tumours or inflammation areas (Naahidi et al. 2013; Al-Obaidi \& Florence 2015; Liu et al. 2016). During navigation through the blood stream, but especially during uptake by a living cell via endocytosis (Doherty \& McMahon 2009; Meinel

$\dagger$ Email address for correspondence: abdallah.daddi-moussa-ider@uni-bayreuth.de $\$$ These authors contributed equally to this work. 
et al. 2014; Agudo-Canalejo \& Lipowsky 2015), nanoparticles frequently come into close contact with cell membranes which alter their hydrodynamic mobilities in a complex fashion.

Over the last few decades, considerable research effort has been devoted to study the motion of particles in the vicinity of interfaces. The particularly simple example of a solid spherical particle has been extensively studied theoretically near a rigid no-slip wall (Lorentz 1907; Brenner 1961; Goldman, Cox \& Brenner 1967a,b; Cichocki \& Jones 1998; Swan \& Brady 2007; Franosch \& Jeney 2009; Happel \& Brenner 2012), an interface separating two immiscible liquids (Lee, Chadwick \& Leal 1979; Bickel 2006, 2007; Wang, Prabhakar \& Sevick 2009; Bławzdziewicz, Ekiel-Jeżewska \& Wajnryb 2010; Bickel 2014), an interface with partial slip (Lauga \& Squires 2005; Felderhof 2012) and a membrane with surface elasticity (Felderhof 2006a,b; Shlomovitz et al. 2013, 2014; Salez \& Mahadevan 2015; Daddi-Moussa-Ider, Guckenberger \& Gekle 2016a,b; Daddi-Moussa-Ider \& Gekle 2016; Saintyves et al. 2016). Elastic membranes stand apart from both liquid-solid and liquid-liquid interfaces, since the elasticity of the membrane introduces a memory effect in the system causing, e.g. anomalous diffusion (Daddi-Moussa-Ider et al. 2016a) or a sign reversal of two-particle hydrodynamic interactions (Daddi-Moussa-Ider \& Gekle 2016). On the experimental side, the near-wall mobility of a spherical particle has been investigated using optical tweezers (Faucheux \& Libchaber 1994; Lin, Yu \& Rice 2000; Dufresne, Altman \& Grier 2001; Schäffer, Nørrelykke \& Howard 2007), digital video microscopy (Eral et al. 2010; Cervantes-Martínez et al. 2011; Dettmer et al. 2014; Tränkle, Ruh \& Rohrbach 2016) and evanescent wave dynamic light scattering (Holmqvist, Dhont \& Lang 2007; Michailidou et al. 2009; Lisicki et al. 2012; Rogers et al. 2012; Michailidou et al. 2013; Lisicki et al. 2014; Wang \& Huang 2014), where a significant alteration of particle motion has been observed in line with theoretical predictions. The influence of a nearby elastic cell membrane has been further investigated using optical traps (Kress et al. 2005; Shlomovitz et al. 2013; Boatwright et al. 2014; Jünger et al. 2015) and magnetic particle actuation (Irmscher et al. 2012).

Particles with a non-spherical shape, such as spheroids or rod-like particles, have also received researchers' attention. The first attempt to investigate the Brownian motion of an anisotropic particle dates back to Perrin $(1934,1936)$ who computed analytically the drag coefficients for a spheroid diffusing in a bulk fluid. A few decades later, Batchelor (1970) pioneered the idea that the flow field surrounding a slender body, such as an elongated particle, may conveniently be represented by a line distribution of Stokeslets between the foci. The method has successfully been applied to a wide range of external flows (Chwang \& Wu 1975) and near boundaries such as a plane hard wall (De Mestre \& Russel 1975; Schiby \& Gallily 1980; Mitchell \& Spagnolie 2015) or a fluid-fluid interface (Blake \& Fulford 1981). Using the multipole expansion of the near-wall flow field, Lisicki, Cichocki \& Wajnryb (2016) have shown that to leading order the mobility of an arbitrary axisymmetric particle near a hard wall can be expressed in closed form by combining the appropriate Green's function with the particle's bulk mobility. Direct simulation numerical investigations of colloidal axisymmetric particles near a wall have been carried out using boundary integral methods (Hsu \& Ganatos 1989), stochastic rotation dynamics (Padding \& Briels 2010; Neild et al. 2010) and finite element methods (De Corato et al. 2015).

Diffusion of micrometre-sized ellipsoidal particles has been investigated experimentally using digital video microscopy (Han et al. 2006, 2009; Neild et al. 2010; Zheng \& Han 2010). Experiments on actin filaments have been conducted using 
fluorescence imaging and particle tracking (Li \& Tang 2004) finding that the measured diffusion coefficients can appropriately be accounted for by a correction resting on the hydrodynamic theory of a long cylinder confined between two walls. The confined rotational diffusion coefficients of carbon nanotubes have been measured using fluorescence video microscopy (Duggal \& Pasquali 2006) and optical microscopy (Bhaduri, Neild \& Ng 2008), where a reasonable agreement has been reported with theoretical predictions. More recently, the three-dimensional rotational diffusion of nanorods (Cheong \& Grier 2010) and rod-like colloids have been measured using video (Colin et al. 2012) and confocal microscopy (Mukhija \& Solomon 2007).

Yet, to the best of our knowledge, motion of a non-spherical particle in the vicinity of deformable elastic interfaces has not been studied so far. In this contribution, we examine the dynamics of an axisymmetric particle near a red blood cell (RBC) membrane using theoretical predictions in close combination with fully resolved boundary integral simulations. The results of the present theory may be used in microrheology experiments in order to characterise the mechanical properties of the membrane (Waigh 2016).

The paper is organised as follows. In $\S 2$, we formulate the theoretical framework for the description of the motion of a colloidal particle in the vicinity of an elastic membrane. We introduce the notion of hydrodynamic friction, mobility and a model for the membrane. In $\S 3$ we outline the mathematical derivation of the correction to the bulk mobility tensor of the particle due to the presence of an interface and provide explicit expressions for the correction valid for any axially symmetric particle. In $\S 4$, we describe the boundary integral method (BIM) used to numerically compute the components of the mobility tensor. Section 5 contains a comparison of analytical predictions and numerical simulations for a spheroidal particle, followed by concluding remarks in $\S 6$. The mathematical details arising in the course of the work are discussed in the appendices.

\section{Hydrodynamics near a membrane}

We consider an axially symmetric particle immersed in an incompressible Newtonian fluid, moving close to an elastic membrane. The fluid is assumed to have the same dynamic viscosity $\eta$ on both sides of the membrane. As an example, we will focus later on a prolate spheroidal particle as shown in figure 1 . The position of the centre of the particle is $\boldsymbol{r}_{0}$, while its orientation is described by the unit vector $\boldsymbol{u}_{1}$ pointing along the symmetry axis. The laboratory frame is spanned by the basis vectors $\left\{\boldsymbol{e}_{x}, \boldsymbol{e}_{y}, \boldsymbol{e}_{z}\right\}$.

We denote by $z_{0}$ the vertical distance separating the centre of the particle from the undisplaced membrane located at the plane $z=0$ and extended infinitely in the horizontal plane $x y$. It is convenient to introduce the body-fixed frame of reference, formed by the three basis vectors $\left\{\boldsymbol{u}_{1}, \boldsymbol{u}_{2}, \boldsymbol{u}_{3}\right\}$. The unit vector $\boldsymbol{u}_{2}$ is parallel to the undisplaced membrane and perpendicular to the particle axis, and $\boldsymbol{u}_{3}$ completes the orthonormal basis. We define $\theta$ as the angle between $\boldsymbol{u}_{1}$ and $\boldsymbol{e}_{z}$ such that $\cos \theta=\boldsymbol{e}_{z} \cdot \boldsymbol{u}_{1}$. The basis vectors in the particle frame are then given by $\boldsymbol{u}_{2}=\left(\boldsymbol{e}_{z} \times \boldsymbol{u}_{1}\right) /\left|\boldsymbol{e}_{z} \times \boldsymbol{u}_{1}\right|$ and $\boldsymbol{u}_{3}=\boldsymbol{u}_{1} \times \boldsymbol{u}_{2}$.

In the inertia-free regime of motion, the fluid dynamics is governed by the stationary incompressible Stokes equations

$$
\begin{aligned}
\eta \nabla^{2} \boldsymbol{v}(\boldsymbol{r})-\nabla p(\boldsymbol{r})+\boldsymbol{f}(\boldsymbol{r}) & =0, \\
\nabla \cdot \boldsymbol{v}(\boldsymbol{r}) & =0,
\end{aligned}
$$




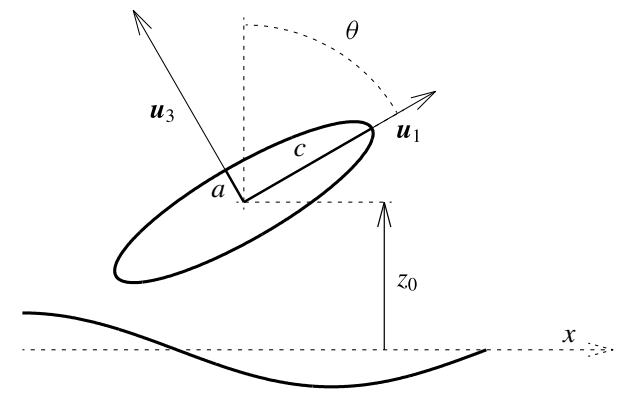

FIGURE 1. Illustration of a spheroidal particle located at $z=z_{0}$ above an elastic cell membrane. The short and long axes are denoted by $a$ and $c$, respectively. The unit vector $\boldsymbol{u}_{1}$ is pointing along the spheroid symmetry axis and $\boldsymbol{u}_{2}$ is the unit vector perpendicular to the plane of the figure. The unit vector $\boldsymbol{u}_{3}$ is defined to be orthogonal to both $\boldsymbol{u}_{1}$ and $u_{2}$.

where $v$ is the fluid velocity, $p$ is the pressure field and $f$ is the force density acting on the fluid due to the presence of the particle. We omit the unsteady term in the Stokes equations, since in realistic situations it leads to a negligible contribution to the mobility corrections (Daddi-Moussa-Ider et al. 2016a). For a discussion accounting for the unsteady term in the bulk flow, see recent work by Felderhof (2013). The flow $\boldsymbol{v}(\boldsymbol{r})$ may be superposed with an arbitrary external flow $\boldsymbol{v}_{0}(\boldsymbol{r})$ being a solution to the homogeneous Stokes equations in the absence of the particle.

Consider now a colloidal particle near the membrane. The total force $\boldsymbol{F}$, torque $\boldsymbol{T}$ and stresslet (symmetric force dipole) $\boldsymbol{S}$ are linearly related to the velocities (translational $\boldsymbol{V}$ and angular $\boldsymbol{\Omega}$ ) of the particle relative to an external flow by the generalised friction tensor (Kim \& Karrila 2013)

$$
\left(\begin{array}{c}
\boldsymbol{F} \\
\boldsymbol{T} \\
\boldsymbol{S}
\end{array}\right)=\left(\begin{array}{lll}
\zeta^{t t} & \zeta^{t r} & \zeta^{t d} \\
\zeta^{r t} & \zeta^{r r} & \zeta^{r d} \\
\zeta^{d t} & \zeta^{d r} & \zeta^{d d}
\end{array}\right)\left(\begin{array}{c}
\boldsymbol{v}_{0}-\boldsymbol{V} \\
\omega_{0}-\boldsymbol{\Omega} \\
\boldsymbol{E}_{0}
\end{array}\right)
$$

with $\boldsymbol{v}_{0}=\boldsymbol{v}_{0}\left(\boldsymbol{r}_{0}\right)$, the vorticity $\boldsymbol{\omega}_{0}=(1 / 2) \nabla \times \boldsymbol{v}_{0}\left(\boldsymbol{r}_{0}\right)$ and the rate of strain $\boldsymbol{E}_{0}=\overline{\nabla \boldsymbol{v}_{0}}\left(\boldsymbol{r}_{0}\right)$ of the external flow (the bar denotes the symmetric and traceless part of the velocity gradient).

A complementary relation defines the generalised mobility tensor

$$
\left(\begin{array}{c}
\boldsymbol{V}-\boldsymbol{v}_{0} \\
\boldsymbol{\Omega}-\boldsymbol{\omega}_{0} \\
-\boldsymbol{S}
\end{array}\right)=\left(\begin{array}{lll}
\boldsymbol{\mu}^{t t} & \boldsymbol{\mu}^{t r} & \boldsymbol{\mu}^{t d} \\
\boldsymbol{\mu}^{r t} & \boldsymbol{\mu}^{r r} & \boldsymbol{\mu}^{r d} \\
\boldsymbol{\mu}^{d t} & \boldsymbol{\mu}^{d r} & \boldsymbol{\mu}^{d d}
\end{array}\right)\left(\begin{array}{c}
\boldsymbol{F} \\
\boldsymbol{T} \\
\boldsymbol{E}_{0}
\end{array}\right)
$$

Upon examining (2.3) and (2.4), we note that the $6 \times 6$ mobility tensor $\boldsymbol{\mu}$ is the inverse of the friction tensor $\zeta$

$$
\zeta^{-1}=\left(\begin{array}{ll}
\zeta^{t t} & \zeta^{t r} \\
\zeta^{r t} & \zeta^{r r}
\end{array}\right)^{-1}=\left(\begin{array}{ll}
\boldsymbol{\mu}^{t t} & \boldsymbol{\mu}^{t r} \\
\boldsymbol{\mu}^{r t} & \boldsymbol{\mu}^{r r}
\end{array}\right)=\boldsymbol{\mu}
$$

Relations between other elements of the generalised mobility and friction tensors may be found directly from (2.3) and (2.4). These are general properties of Stokes 
flows following from the linearity of the governing equations. Finding an explicit form of these tensors requires the solution of Stokes equations (2.1) and (2.2) with appropriate boundary conditions on the confining interfaces. Since we aim at computing the particle mobility nearby a membrane endowed with surface elasticity and bending resistance, a relevant model for the membrane dynamics needs to be introduced at this point.

The Skalak model (Skalak et al. 1973) is well established and commonly used to represent RBC membranes (Freund 2014). The elastic properties of the interface are characterised by two moduli: elastic shear modulus $\kappa_{S}$ and area dilatation modulus $\kappa_{A}$. Resistance towards bending has been further included following the model of Helfrich (1973) with the associated bending modulus $\kappa_{B}$. In this approach, the linearised tangential and normal traction jumps across the membrane are related to the membrane displacement field $\boldsymbol{h}$ at $z=0$ and the dilatation $\epsilon$ by (Daddi-Moussa-Ider et al. 2016a)

$$
\begin{aligned}
& {\left[\sigma_{z \alpha}\right]=-\frac{\kappa_{S}}{3}\left(\Delta_{\|} h_{\alpha}+(1+2 C) \partial_{\alpha} \epsilon\right), \quad \alpha \in\{x, y\},} \\
& {\left[\sigma_{z z}\right]=\kappa_{B} \Delta_{\|}^{2} h_{z}}
\end{aligned}
$$

where $[f]:=f\left(z=0^{+}\right)-f\left(z=0^{-}\right)$denotes the jump of the quantity $f$ across the membrane. The dilatation $\epsilon:=\partial_{x} h_{x}+\partial_{y} h_{y}$ is the trace of the strain tensor. The Skalak parameter is defined as $C:=\kappa_{A} / \kappa_{S}$. Here $\Delta_{\|}:=\partial_{x}^{2}+\partial_{y}^{2}$ is the Laplace-Beltrami operator along the membrane. The components $\sigma_{z \alpha}$ of the stress tensor in the fluid are expressed in a standard way by $\sigma_{z \alpha}=-p \delta_{z \alpha}+\eta\left(\partial_{\alpha} v_{z}+\partial_{z} v_{\alpha}\right)$ for $\alpha \in\{x, y, z\}$ (Kim \& Karrila 2013).

The membrane displacement $\boldsymbol{h}$ and the fluid velocity $\boldsymbol{v}$ are related by the no-slip boundary condition at the undisplaced membrane, which in Fourier space takes the form

$$
v_{\alpha}=\left.\mathrm{i} \omega h_{\alpha}\right|_{z=0}, \quad \alpha \in\{x, y, z\},
$$

with $\omega$ being the characteristic frequency of forcing in the system. The frequencydependent elastic deformation effects are characterised by two dimensionless parameters, as described in Daddi-Moussa-Ider et al. (2016a)

$$
\beta=\frac{12 z_{0} \eta \omega}{\kappa_{S}+\kappa_{A}}, \quad \beta_{B}=2 z_{0}\left(\frac{4 \eta \omega}{\kappa_{B}}\right)^{1 / 3},
$$

Further details of the derivation of $\beta$ and $\beta_{B}$ can be found in appendix A. The effects of shear resistance and area dilatation are thus captured by $\beta$, while $\beta_{B}$ describes the bending resistance of the membrane. In the steady case for which $\beta=\beta_{B}=0$, corresponding to a vanishing frequency or to an infinitely stiff membrane, we expect to recover the results for a hard no-slip wall.

In the case of periodic forcing or time-dependent deformation of the membrane, the quantity of interest is the frequency-dependent mobility tensor. Our aim in this work is to find all the components of $\boldsymbol{\mu}(\omega)$ for an axisymmetric particle close to an elastic membrane. Accordingly, due to the presence of the interface, the near-membrane mobility will then have a correction on top of the bulk mobility $\boldsymbol{\mu}_{0}$,

$$
\boldsymbol{\mu}(\omega)=\boldsymbol{\mu}_{0}+\boldsymbol{\Delta} \boldsymbol{\mu}(\omega)
$$


stemming from the interaction of the flow created by the particle with the boundary. To determine the form of $\boldsymbol{\mu}(\omega)$ in an approximate manner, we use the results by Lisicki et al. (2016) valid for a hard no-slip wall and generalize them to the case of an elastic membrane. Their idea is based on a multipole expansion (Cichocki et al. 2000) of the flow field around an axially symmetric particle close to a boundary, with a corresponding expansion of the force distribution on its surface. If the particle is sufficiently far away from the wall, they have shown that the dominant correction to its friction matrix can be viewed as an interaction between the centre of the particle and its hydrodynamic image. They provide explicit expressions for the elements of the friction tensor for all types of motion (translation, rotation and coupling terms) which yields the corrected mobility tensor upon inversion. The same route may be followed for a membrane, provided that the form of the Green's tensor for the system is known.

A general Stokes flow can be constructed using the Green's function $\mathcal{G}\left(\boldsymbol{r}, \boldsymbol{r}^{\prime}\right)$ being the solution of (2.1) and (2.2) subject to a time-dependent point force $\boldsymbol{f}(r)=\boldsymbol{F}(t) \delta(\boldsymbol{r}-$ $\left.\boldsymbol{r}^{\prime}\right)$ with the appropriate boundary conditions on the membrane. In an unbounded fluid, the Green's tensor is the Oseen tensor (Kim \& Karrila 2013) $\mathcal{G}_{0}\left(\boldsymbol{r}, \boldsymbol{r}^{\prime}\right)=\mathcal{G}_{0}\left(\boldsymbol{r}-\boldsymbol{r}^{\prime}\right)$, with $\mathcal{G}_{0}(\boldsymbol{r})=\left(\mathbf{1}+\boldsymbol{r} \boldsymbol{r} / r^{2}\right) /(8 \pi \eta r)$ and $r:=|\boldsymbol{r}|$. In the presence of boundaries, the Green's tensor contains the extra term $\Delta \mathcal{G}$ describing the flow reflected from the membrane, so that $\mathcal{G}=\mathcal{G}_{0}+\Delta \mathcal{G}$.

The exact Green's function for a point force close to a membrane has recently been computed in Daddi-Moussa-Ider et al. (2016a). For the resolution of (2.1) and (2.2) with a point force acting at $\boldsymbol{r}_{0}=\left(0,0, z_{0}\right)$, the two-dimensional Fourier transform in the $x y$ plane was used to solve the resulting equations with accordingly transformed boundary conditions. The procedure has been previously described in detail and therefore we only list the main steps for the determination of the Green's tensor in appendix A of this work.

\section{Near-membrane mobility tensors}

We search for the near-membrane mobility tensor, $\boldsymbol{\mu}(\omega)=\boldsymbol{\mu}_{0}+\boldsymbol{\Delta} \boldsymbol{\mu}(\omega)$ by calculating the leading-order correction to the bulk mobility. To this end, we follow the route outlined in a recent contribution by Lisicki et al. (2016) who derived analytic expressions for the friction tensor of an axially symmetric particle in the presence of a hard no-slip wall. The friction tensor, similarly to the mobility tensor, can be split into the bulk and the correction term

$$
\zeta=\zeta_{0}+\Delta \zeta
$$

The final expressions for the corrected friction tensor involve elements of the bulk friction tensor of the particle, and the distance- and orientation-dependent (derivatives of) the appropriate Green's function. For the hard no-slip wall treated in Lisicki et al. (2016) the latter is the Blake tensor (Blake 1971) while in the present case the frequency-dependent Green's functions from Daddi-Moussa-Ider et al. (2016a) are employed. The expressions for the friction tensor with a general Green's function read (Lisicki et al. 2016)

$$
\begin{aligned}
\Delta \zeta^{t t} & =-\frac{1}{8 \pi \eta} \frac{1}{2 z_{0}} \zeta_{0}^{t t} \boldsymbol{g}^{t t} \zeta_{0}^{t t}+\frac{1}{(8 \pi \eta)^{2}} \frac{1}{\left(2 z_{0}\right)^{2}} \boldsymbol{\zeta}_{0}^{t t} \boldsymbol{g}^{t t} \boldsymbol{\zeta}_{0}^{t t} \boldsymbol{g}^{t t} \boldsymbol{\zeta}_{0}^{t t}+O\left(z_{0}^{-3}\right), \\
\Delta \boldsymbol{\zeta}^{t r} & =-\frac{1}{8 \pi \eta} \frac{1}{\left(2 z_{0}\right)^{2}} \boldsymbol{\zeta}_{0}^{t t} \boldsymbol{g}^{t d} \boldsymbol{\zeta}_{0}^{d r}+O\left(z_{0}^{-3}\right),
\end{aligned}
$$




$$
\begin{aligned}
& \boldsymbol{\Delta} \boldsymbol{\zeta}^{r t}=-\frac{1}{8 \pi \eta} \frac{1}{\left(2 z_{0}\right)^{2}} \zeta_{0}^{r d} \boldsymbol{g}^{d t} \boldsymbol{\zeta}_{0}^{t t}+O\left(z_{0}^{-3}\right), \\
& \boldsymbol{\Delta} \boldsymbol{\zeta}^{r r}=-\frac{1}{8 \pi \eta} \frac{1}{\left(2 z_{0}\right)^{3}}\left[\zeta_{0}^{r r} \boldsymbol{g}^{r r} \boldsymbol{\zeta}_{0}^{r r}+\boldsymbol{\zeta}_{0}^{r r} \boldsymbol{g}^{r d} \boldsymbol{\zeta}_{0}^{d r}+\zeta_{0}^{r d} \boldsymbol{g}^{d r} \boldsymbol{\zeta}_{0}^{r r}+\boldsymbol{\zeta}_{0}^{r d} \boldsymbol{g}^{d d} \zeta_{0}^{d r}\right]+O\left(z_{0}^{-4}\right),
\end{aligned}
$$

where the directional tensors $\boldsymbol{g}$ are defined by

$$
\Delta \mathcal{G}^{\gamma \delta}=\frac{1}{8 \pi \eta} \frac{1}{\left(2 z_{0}\right)^{a}} \boldsymbol{g}^{\gamma \delta}
$$

Here, $\boldsymbol{\Delta} \mathcal{G}^{\gamma \delta}$ are the multipole elements of the Green's integral operator which will be derived below. Further, $\gamma, \delta \in\{t, r, d\}$ and $a=1$ for $t t, a=2$ for $(t r, r t, t d, d t)$ and $a=3$ for $(d r, r d, r r, d d)$ parts. In (3.2)-(3.5) it should be understood that the tensors are appropriately contracted to yield second-order tensor corrections.

We now apply this result to our system. Our goal is to obtain explicit expressions for the mobility tensors for an axially symmetric particle in the presence of a membrane in terms of its bulk mobilities. This can be done in two steps.

Firstly, we invert the friction relations (3.2)-(3.5), as detailed in appendix B, to obtain analogous relations for the mobilities:

$$
\begin{aligned}
\Delta \boldsymbol{\mu}^{t t} & =\frac{1}{8 \pi \eta} \frac{1}{2 z_{0}} \boldsymbol{g}^{t t}+O\left(z_{0}^{-3}\right), \\
\boldsymbol{\Delta} \boldsymbol{\mu}^{t r} & =-\frac{1}{8 \pi \eta} \frac{1}{\left(2 z_{0}\right)^{2}} \boldsymbol{g}^{t d} \boldsymbol{\mu}_{0}^{d r}+O\left(z_{0}^{-3}\right), \\
\boldsymbol{\Delta} \boldsymbol{\mu}^{r t} & =\frac{1}{8 \pi \eta} \frac{1}{\left(2 z_{0}\right)^{2}} \boldsymbol{\mu}_{0}^{r d} \boldsymbol{g}^{d t}+O\left(z_{0}^{-3}\right), \\
\boldsymbol{\Delta} \boldsymbol{\mu}^{r r} & =\frac{1}{8 \pi \eta} \frac{1}{\left(2 z_{0}\right)^{3}}\left[\boldsymbol{g}^{r r}-\boldsymbol{\mu}_{0}^{r d} \boldsymbol{g}^{d r}+\boldsymbol{g}^{r d} \boldsymbol{\mu}_{0}^{d r}-\boldsymbol{\mu}_{0}^{r d} \boldsymbol{g}^{d d} \boldsymbol{\mu}_{0}^{d r}\right]+O\left(z_{0}^{-4}\right) .
\end{aligned}
$$

These expressions allow straightforward computation of the near-membrane mobilities for axisymmetric particles of arbitrary shape if their bulk mobilities are known, either numerically or analytically. Compared to a numerical inversion of the friction tensor, which in principle would be preferable as it avoids the possibility of negative mobilities (Lisicki et al. 2016), this approach has the advantage that explicit analytical expressions for the mobility can be obtained.

Remarkably, the final formulae include only one bulk characteristic of the particle, namely the tensors $\boldsymbol{\mu}_{0}^{r d}$ and $\boldsymbol{\mu}_{0}^{d r}$ which describe the rotational motion of the particle in response to elongational flow. This form follows from the particular symmetries of an axially symmetric particle with inversional symmetry $\left(\boldsymbol{u}_{1} \leftrightarrow-\boldsymbol{u}_{1}\right)$.

Secondly, to obtain the directional tensors $\boldsymbol{g}$, we consider a general Green's tensor $\mathcal{G}\left(\boldsymbol{r}, \boldsymbol{r}^{\prime}\right)=\mathcal{G}_{0}\left(\boldsymbol{r}-\boldsymbol{r}^{\prime}\right)+\boldsymbol{\Delta} \mathcal{G}\left(\boldsymbol{r}, \boldsymbol{r}^{\prime}\right)$ and a body placed at $\boldsymbol{r}_{0}$ with a force distribution $\boldsymbol{f}(\boldsymbol{r})$ on its surface. The flow at a point $r$ due to this forcing may be written as the integral equation

$$
\boldsymbol{v}(\boldsymbol{r})=\int \mathrm{d} \boldsymbol{r}^{\prime} \mathcal{G}\left(\boldsymbol{r}, \boldsymbol{r}^{\prime}\right) \cdot \boldsymbol{f}\left(\boldsymbol{r}^{\prime}\right),
$$

with the integral performed over the surface of the body. The idea of the derivation of the correction is to find, given the force density, the flow incident on the particle 
itself due to the presence of an interface. Thus we consider (3.11) with only the membrane interaction part $\Delta \mathcal{G}\left(\boldsymbol{r}, \boldsymbol{r}^{\prime}\right)$ of the Green's tensor and expand it in both arguments around $\boldsymbol{r}=\boldsymbol{r}^{\prime}=\boldsymbol{r}_{0}$. The integrals of the subsequent terms on the right-hand side reproduce the force multipole moments, while the expansion of the left-hand side yields the multipole expansion of the flow field. By matching the relevant multipoles, we find explicit expressions for the $\boldsymbol{\Delta} \mathcal{G}^{\gamma \delta}$, with $\gamma, \delta \in\{t, r, d\}$, as described in Lisicki et al. (2016). The resulting formulae are

$$
\begin{aligned}
\Delta \mathcal{G}_{\alpha \beta}^{t t} & =\lim _{r, r^{\prime} \rightarrow r_{0}} \Delta \mathcal{G}_{\alpha \beta}, \\
\Delta \mathcal{G}_{\alpha \beta}^{t r} & =\lim _{r, r^{\prime} \rightarrow r_{0}} \frac{1}{2} \epsilon_{\alpha \mu \nu} \partial_{\mu} \Delta \mathcal{G}_{\nu \beta}, \\
\Delta \mathcal{G}_{\alpha \beta}^{r t} & =\lim _{r, r^{\prime} \rightarrow r_{0}}-\frac{1}{2} \epsilon_{\mu \nu \beta} \partial_{\nu}^{\prime} \Delta \mathcal{G}_{\alpha \mu}, \\
\Delta \mathcal{G}_{\alpha \beta}^{r r} & =\lim _{r, r^{\prime} \rightarrow r_{0}} \frac{1}{4} \epsilon_{\alpha \mu \gamma} \epsilon_{\beta \nu \eta} \partial_{\gamma} \partial_{\eta}^{\prime} \Delta \mathcal{G}_{\mu \nu}, \\
\Delta \mathcal{G}_{\alpha \beta \gamma}^{t d} & =\lim _{r, r^{\prime} \rightarrow r_{0}} \partial_{\gamma}^{\prime} \Delta \mathcal{G}_{\alpha \beta}, \\
\Delta \mathcal{G}_{\alpha \beta \gamma}^{d t} & =\lim _{r, r^{\prime} \rightarrow r_{0}} \partial_{\alpha} \Delta \mathcal{G}_{\beta \gamma}, \\
\Delta \mathcal{G}_{\alpha \beta \gamma}^{d r} & =\lim _{r, r^{\prime} \rightarrow r_{0}}-\frac{1}{2} \epsilon_{\gamma \mu \nu}{ }^{\prime} \overbrace{\nu} \partial_{\alpha} \Delta \mathcal{G}_{\beta \mu} \\
\Delta \mathcal{G}_{\alpha \beta \gamma}^{r d} & =\lim _{r, r^{\prime} \rightarrow r_{0}} \frac{1}{2} \epsilon_{\alpha \mu \nu} \partial_{\mu}^{\prime} \overbrace{\beta} \Delta \mathcal{G}_{\nu \gamma}^{(\beta \gamma)}, \\
\Delta \mathcal{G}_{\alpha \beta \gamma \delta}^{d d} & =\lim _{r, r^{\prime} \rightarrow r_{0}}{ }_{\partial_{\alpha} \partial_{\delta}^{\prime} \Delta \mathcal{G}_{\beta \gamma}}^{(\alpha \beta)(\gamma \delta)},
\end{aligned}
$$

where $\epsilon_{\alpha \mu \nu}$ is the Levi-Civita tensor and the symbol $\Gamma^{(\alpha \beta)}$ denotes the symmetric and traceless part with respect to indices $\alpha, \beta$. Explicitly, the reductions for an arbitrary third- and fourth-order traceless tensor read

$$
\begin{aligned}
& {\overparen{M_{\alpha \beta \gamma}}}^{(\alpha \beta)}=\frac{1}{2}\left(M_{\alpha \beta \gamma}+M_{\beta \alpha \gamma}\right), \\
& \overparen{M}_{\alpha \beta \gamma \delta}^{(\alpha \beta)(\gamma \delta)}=\frac{1}{4}\left(M_{\alpha \beta \gamma \delta}+M_{\beta \alpha \gamma \delta}+M_{\alpha \beta \delta \gamma}+M_{\beta \alpha \delta \gamma}\right) \text {. }
\end{aligned}
$$

The prime denotes a derivative with respect to the second argument. We note that the tensors $\Delta \mathcal{G}^{d r, r d, d d}$ are traceless due to the incompressibility of the fluid, and therefore the trace need not be subtracted in the procedure of symmetrisation. We further remark that (3.12) through (3.20) involves differentiations and elementary operations that are well defined for complex quantities, and hence lead to convergent limits.

It is most natural to consider the correction in the reference frame of the particle, spanned by the three unit basis vectors $\left\{\boldsymbol{u}_{1}, \boldsymbol{u}_{2}, \boldsymbol{u}_{3}\right\}$. In this frame, the mobility tensors of an axisymmetric particle have the form

$$
\Delta \boldsymbol{\mu}^{t t, r r}=\left(\begin{array}{ccc}
\Delta \mu_{11}^{t t, r r} & 0 & \Delta \mu_{13}^{t t, r r} \\
0 & \Delta \mu_{22}^{t t, r r} & 0 \\
\Delta \mu_{13}^{t t, r r} & 0 & \Delta \mu_{33}^{t t, r r}
\end{array}\right)
$$

for translational and rotational motion, while the translation-rotation coupling tensor reads

$$
\Delta \boldsymbol{\mu}^{t r}=\left(\begin{array}{ccc}
0 & \Delta \mu_{12}^{t r} & 0 \\
0 & 0 & \Delta \mu_{23}^{t r} \\
0 & \Delta \mu_{32}^{t r} & 0
\end{array}\right) .
$$


The rotation-translation coupling tensor $\Delta \boldsymbol{\mu}^{r t}$ is obtained by simply taking the transpose of the translation-rotation coupling tensor given above. (See supplemental material available at https://doi.org/10.1017/jfm.2016.739 for the frequency-dependent mobility corrections expressed in the laboratory frame).

\section{Boundary integral method}

Here we introduce the boundary integral method (Pozrikidis 2001) used to numerically compute the mobility tensor of a truly extended spheroidal particle. The method is perfectly suited for treating three-dimensional problems with complex, deforming boundaries such as RBC membranes in the Stokes regime (Zhao \& Shaqfeh 2011; Zhu 2014). In order to solve for the particle motion, given an applied force or torque, we combine a completed double layer boundary integral method (CDLBIM) (Power \& Miranda 1987) with the classical BIM (Zhao, Shaqfeh \& Narsimhan 2012). The resulting equations are then discretised and transformed into a system of algebraic equations as detailed in (Daddi-Moussa-Ider et al. 2016b; Guckenberger et al. 2016).

For the numerical determination of the particle mobility components, a harmonic force $\boldsymbol{F}(t)=\boldsymbol{A} \mathrm{e}^{\mathrm{i} \omega_{0} t}$ or torque $\boldsymbol{T}(t)=\boldsymbol{B} \mathrm{e}^{\mathrm{i} \omega_{0} t}$ is applied at the particle surface. After a short transient evolution, the particle linear and angular velocities can be described as $\boldsymbol{V}(t)=\boldsymbol{C} \mathrm{e}^{\mathrm{i}\left(\omega_{0} t+\delta_{t}\right)}$ and $\boldsymbol{\Omega}(t)=\boldsymbol{D} \mathrm{e}^{\mathrm{i}\left(\omega_{0} t+\delta_{r}\right)}$ respectively. The amplitudes and phase shifts can be determined accurately by fitting the numerically recorded velocities using the trust region method (Conn, Gould \& Toint 2000). In the laboratory frame, the components $\mu_{\alpha \beta}^{t t}$ and $\mu_{\alpha \beta}^{r t}$ of the mobility are determined for a torque-free particle as

$$
\mu_{\alpha \beta}^{t t}=\frac{C_{\alpha}}{A_{\beta}} \mathrm{e}^{\mathrm{i} \delta_{t}}, \quad \mu_{\alpha \beta}^{r t}=\frac{D_{\alpha}}{A_{\beta}} \mathrm{e}^{\mathrm{i} \delta_{r}} .
$$

For a force-free particle, the components $\mu_{\alpha \beta}^{t r}$ and $\mu_{\alpha \beta}^{r r}$ are obtained from

$$
\mu_{\alpha \beta}^{t r}=\frac{C_{\alpha}}{B_{\beta}} \mathrm{e}^{\mathrm{i} \delta_{t}}, \quad \mu_{\alpha \beta}^{r r}=\frac{D_{\alpha}}{B_{\beta}} \mathrm{e}^{\mathrm{i} \delta_{r}} .
$$

\section{Spheroid close to a membrane: theoretical and numerical results}

In this section, we present a comparison of our theoretical results to numerical simulations using the example of a prolate spheroidal particle. To begin with, we discuss the bulk mobility of a spheroid. Further on, we show the explicit form of the correction, and finally compare the components of the corrected mobility matrix to numerical simulations.

The bulk translational and rotational mobility tensors of a general axisymmetric particle have the form

$$
\boldsymbol{\mu}_{0}^{t t, r r}=\mu_{\|}^{t, r} \boldsymbol{u}_{1} \boldsymbol{u}_{1}+\mu_{\perp}^{t, r}\left(\mathbf{1}-\boldsymbol{u}_{1} \boldsymbol{u}_{1}\right)
$$

The third-order tensors $\boldsymbol{\mu}_{0}^{r d}$ and $\boldsymbol{\mu}_{0}^{d r}$ have the Cartesian components

$$
\begin{aligned}
& \left(\mu_{0}^{r d}\right)_{\alpha \beta \gamma}=\mu^{r d} u_{\sigma} \overbrace{\sigma \alpha \beta} u_{\gamma}^{(\beta \gamma)}, \\
& \left(\mu_{0}^{d r}\right)_{\alpha \beta \gamma}=\mu^{d r}{\overbrace{u_{\alpha} \epsilon_{\beta \gamma \sigma}}^{(\alpha \beta)}} u_{\sigma},
\end{aligned}
$$


where, following from the Lorentz reciprocal theorem (Kim \& Karrila 2013)

$$
\mu^{d r}=\mu^{r d}=: \lambda
$$

Note that due to the axial and inversional symmetry in bulk, we have $\boldsymbol{\mu}_{0}^{t r}=\boldsymbol{\mu}_{0}^{r t}=0$ and $\boldsymbol{\mu}_{0}^{t d}=\boldsymbol{\mu}_{0}^{d t}=0$.

For a prolate spheroidal particle of eccentricity $e$, analytical results are available and the bulk mobility coefficients are given by Kim \& Karrila (2013, table 3.4)

$$
\begin{aligned}
\mu_{\|}^{t} & =\frac{1}{6 \pi \eta c} \frac{3}{8} \frac{-2 e+\left(1+e^{2}\right) L}{e^{3}}, \\
\mu_{\perp}^{t} & =\frac{1}{6 \pi \eta c} \frac{3}{16} \frac{2 e+\left(3 e^{2}-1\right) L}{e^{3}}, \\
\mu_{\|}^{r} & =\frac{1}{8 \pi \eta c^{3}} \frac{3}{4} \frac{2 e-\left(1-e^{2}\right) L}{e^{3}\left(1-e^{2}\right)}, \\
\mu_{\perp}^{r} & =\frac{1}{8 \pi \eta c^{3}} \frac{3}{4} \frac{-2 e+\left(1+e^{2}\right) L}{e^{3}\left(2-e^{2}\right)},
\end{aligned}
$$

where $a$ and $c$ are the short and long axis of the spheroid and

$$
e=\sqrt{1-\left(\frac{a}{c}\right)^{2}}, \quad L=\ln \left(\frac{1+e}{1-e}\right) .
$$

To obtain the final ingredient $\mu^{r d}$, we observe from the definitions in (2.3) and (2.4) that $\mu_{\alpha \beta \gamma}^{r d}=\mu_{\alpha \delta}^{r r} \zeta_{\delta \beta \gamma}^{r d}$ and $\mu_{\alpha \beta \gamma}^{d r}=-\zeta_{\alpha \beta \delta}^{d r} \mu_{\delta \gamma}^{r r}$, leading to

$$
\mu^{r d}=\mu_{\perp}^{r} \zeta^{r d}
$$

The component $r d$ of the friction tensor is (Kim \& Karrila 2013)

$$
\frac{\zeta^{r d}}{8 \pi \eta c^{3}}=\frac{4}{3} \frac{e^{5}}{-2 e+\left(1+e^{2}\right) L} .
$$

Therefore we obtain the $r d$ coefficient of the mobility tensor

$$
\lambda=\frac{e^{2}}{2-e^{2}} .
$$

Having introduced the bulk hydrodynamic mobilities of a spheroid, we turn our attention to the membrane correction which in the frame of the particle can be written as in (3.23) and (3.24). We find that the corrections to the translational mobilities as given in general form in (3.7) can, for a spheroid, be written in closed form as

$$
\begin{aligned}
& 8 \pi \eta\left(2 z_{0}\right) \Delta \mu_{11}^{t t}=P \sin ^{2} \theta+Q \cos ^{2} \theta, \\
& 8 \pi \eta\left(2 z_{0}\right) \Delta \mu_{13}^{t t}=(P-Q) \sin \theta \cos \theta, \\
& 8 \pi \eta\left(2 z_{0}\right) \Delta \mu_{22}^{t t}=P, \\
& 8 \pi \eta\left(2 z_{0}\right) \Delta \mu_{33}^{t t}=P \cos ^{2} \theta+Q \sin ^{2} \theta,
\end{aligned}
$$


and $\Delta \mu_{13}^{t t}=\Delta \mu_{31}^{t t}$. Thus they have the desired symmetry of (3.23). Expressions for $P\left(\beta, \beta_{B}\right)=P_{S}(\beta)+P_{B}\left(\beta_{B}\right)$ and $Q\left(\beta, \beta_{B}\right)=Q_{S}(\beta)+Q_{B}\left(\beta_{B}\right)$ are provided explicitly in appendix $C$.

For the translation-rotation coupling, the non-vanishing mobility corrections as given by (3.8), can be cast in the frame of the particle as

$$
\begin{aligned}
& 8 \pi \eta\left(2 z_{0}\right)^{2} \Delta \mu_{12}^{t r}=\lambda \sin \theta\left(M+N \cos ^{2} \theta\right), \\
& 8 \pi \eta\left(2 z_{0}\right)^{2} \Delta \mu_{23}^{t r}=\lambda M \cos \theta, \\
& 8 \pi \eta\left(2 z_{0}\right)^{2} \Delta \mu_{32}^{t r}=-\lambda \cos \theta\left(M+N \sin ^{2} \theta\right),
\end{aligned}
$$

where $M$ and $N$ are now functions of the parameters $\beta$ and $\beta_{B}$, and can likewise be decomposed into shearing and bending contributions. The dependence on the bulk $r d$ mobility $\lambda$ is explicitly separated out.

Finally, considering the rotational part as stated by (3.10), the non-vanishing components of the mobility correction in the frame of the particle can conveniently be cast in the following forms

$$
\begin{aligned}
& 8 \pi \eta\left(2 z_{0}\right)^{3} \Delta \mu_{11}^{r r}=A_{0}+A_{2} \cos ^{2} \theta, \\
& 8 \pi \eta\left(2 z_{0}\right)^{3} \Delta \mu_{13}^{r r}=D \sin \theta \cos \theta, \\
& 8 \pi \eta\left(2 z_{0}\right)^{3} \Delta \mu_{22}^{r r}=C_{0}+\lambda C_{2} \cos ^{2} \theta+\lambda^{2} C_{4} \cos ^{4} \theta, \\
& 8 \pi \eta\left(2 z_{0}\right)^{3} \Delta \mu_{33}^{r r}=H_{0}+H_{2} \cos ^{2} \theta,
\end{aligned}
$$

and with $\Delta \mu_{13}^{r r}=\Delta \mu_{31}^{r r}$. All the functions depend on $\left(\beta, \beta_{B}\right)$ and are decomposed into bending and shearing parts in appendix $C$. In addition, the functions $C, D$ and $H$ depend on the coefficient $\lambda$.

It can be seen that the mobility corrections for an axisymmetric particle in their dominant terms possess a simple angular structure. The latter stems from the contraction of the particle friction tensors (which have an axial symmetry, dictated by their shape, with respect to the body axis) with the vertical multipole components of the Blake tensor (which have the same structure but with respect to a different axis, i.e. the vertical direction). This contraction requires transformation of corresponding tensors into the common frame of reference, which generates simple polynomials in sine and cosine functions of the inclination angle as discussed in Lisicki et al. (2016).

In the following, we shall present a comparison between these analytical predictions and numerical simulations using the BIM, presented in $\S 4$. We consider a prolate spheroid of aspect ratio $p:=c / a=2$, inclined at an angle $\theta=\pi / 3$ to the $z$ axis, positioned at $z_{0}=2 c$ above a planar elastic membrane. For the membrane, we take a reduced bending modulus $E_{B}:=c^{2} \kappa_{S} / \kappa_{B}=3 / 2$ for which the characteristic frequencies $\beta$ and $\beta_{B}^{3}$ have the same order of magnitude. The Skalak parameter is $C=1$. Corresponding data showing the effect of the inclination angle and the reduced bending modulus can be found in the Supporting Information. Our analytical predictions are applicable for large and moderate membrane-particle distances for which $c / z_{0} \sim O(1)$ where we find good agreement with numerical simulations.

Henceforth, the mobility corrections will be scaled by the associated bulk values. For diagonal terms, we choose the corresponding diagonal elements, namely $\mu_{\|}^{t, r}$ for $\mu_{11}^{t, r}$ and $\mu_{\perp}^{t, r}$ for $\mu_{22,33}^{t, r}$. For non-diagonal terms, we use an appropriate combination of bulk mobilities, that is $\sqrt{\mu_{\|}^{t, r} \mu_{\perp}^{t, r}}$ for translations and rotations. The translation-rotation coupling tensors are scaled by $\sqrt{\mu_{\perp}^{t} \mu_{\perp}^{r}}$. 

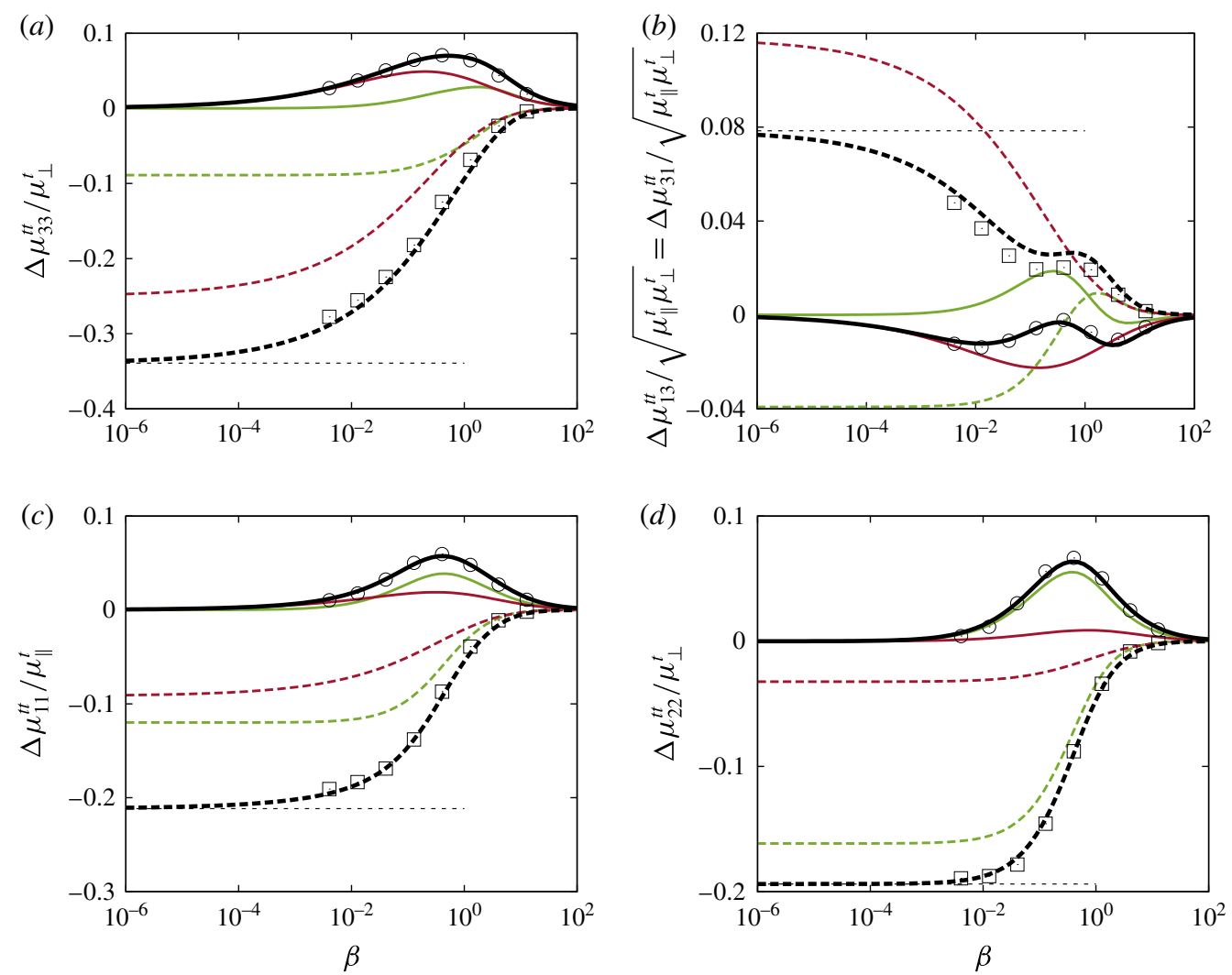

FIGURE 2. (Colour online) The scaled translational mobility correction components versus the scaled frequency. The spheroid is located above the membrane at $z_{0}=2 c$ inclined at an angle $\theta=\pi / 3$ from the vertical. The analytical predictions of the real and imaginary parts of the translational mobility corrections are shown as dashed and solid lines, respectively. The corrections due to shearing and bending are shown respectively in green (bright grey in a black and white printout) and red (dark grey in a black and white printout). Horizontal dotted lines represent the hard-wall limits from Lisicki et al. (2016). BIM simulations are marked as squares and circles for the real and imaginary parts, respectively. For the membrane parameters we take a reduced bending modulus $E_{B}:=\kappa_{B} /\left(c^{2} \kappa_{S}\right)=2 / 3$ and the Skalak parameter $C=1$.

In figure 2 we compare the components of the translational mobility calculated from (5.13)-(5.16) with those obtained from BIM simulations. For the diagonal components we observe that the real part of the complex mobility corrections is monotonically increasing with frequency. The imaginary part exhibits a non-monotonic bell-shaped dependence on frequency that peaks around $\beta \sim 1$. The off-diagonal components 13 and 31 show a more complex dependence on frequency. In the vanishing frequency limit, we recover the corrections near a hard wall with stick boundary conditions recently calculated by Lisicki et al. (2016). We further remark that for the present inclination of $\theta=\pi / 3$ the components 33 and 13 are principally determined by bending resistance whereas the shearing effect is more pronounced in the components 11 and 22. A very good agreement is obtained between analytical 
(a)

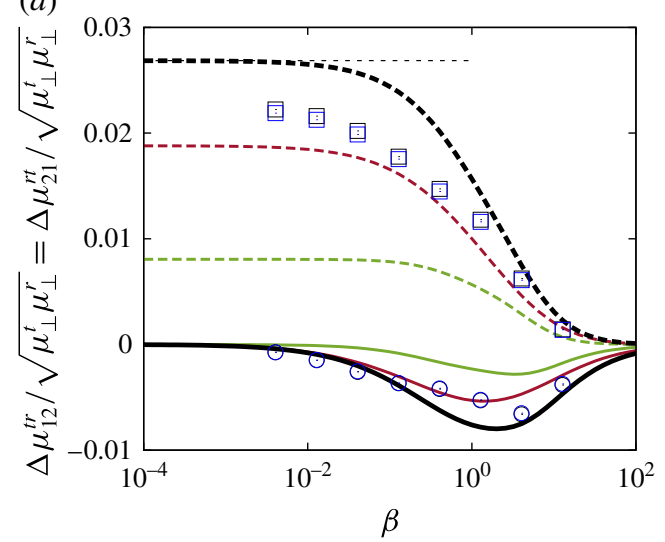

(b) $\quad\left(\times 10^{-3}\right)$

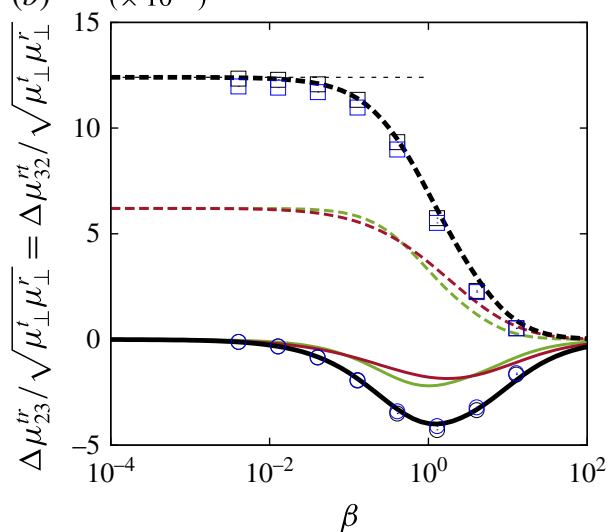

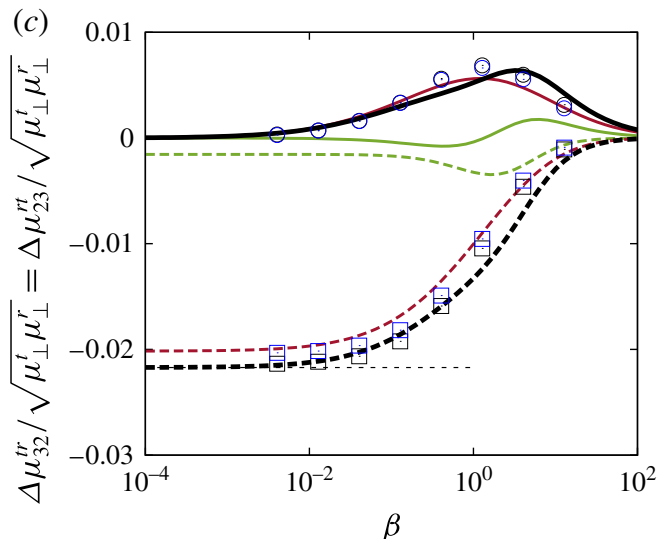

FIGURE 3. (Colour online) The scaled coupling mobility corrections versus the scaled frequency. Black and blue symbols refer to the $t r$ and $r t$ components, respectively, obtained from BIM simulations. The other colours are the same as in figure 2 .

predictions and numerical simulations for all components over the entire range of frequencies.

By examining the off-diagonal component 31 shown in figure $2(b)$, it is clear that the shearing- and bending-related parts may have opposite contributions to the total translational mobility. This observed trend implies that upon exerting a force along $\boldsymbol{u}_{1}$, there exists a drift motion along $\boldsymbol{u}_{3}$, either away or towards the membrane, depending on the shearing and bending properties. In fact, for a membrane with bending-only resistance, such as a fluid vesicle, the spheroid is pushed away from the membrane in the same way as near a hard wall. On the other hand, for a membrane with shearing-only resistance, such as an artificial capsule, the motion is directed towards the membrane.

Figure 3 shows the corrections to the translation-rotation coupling mobility versus the scaled frequency computed from (5.17) and (5.19). We observe that bending resistance is essentially the dominant contributor to the coupling mobility. It can be shown that this trend is always the case regardless of spheroid orientation. The BIM simulation results are consistent with the fact that the $t r$ and $r t$ mobility tensors are the transpose of each other and a good agreement is obtained between analytical 

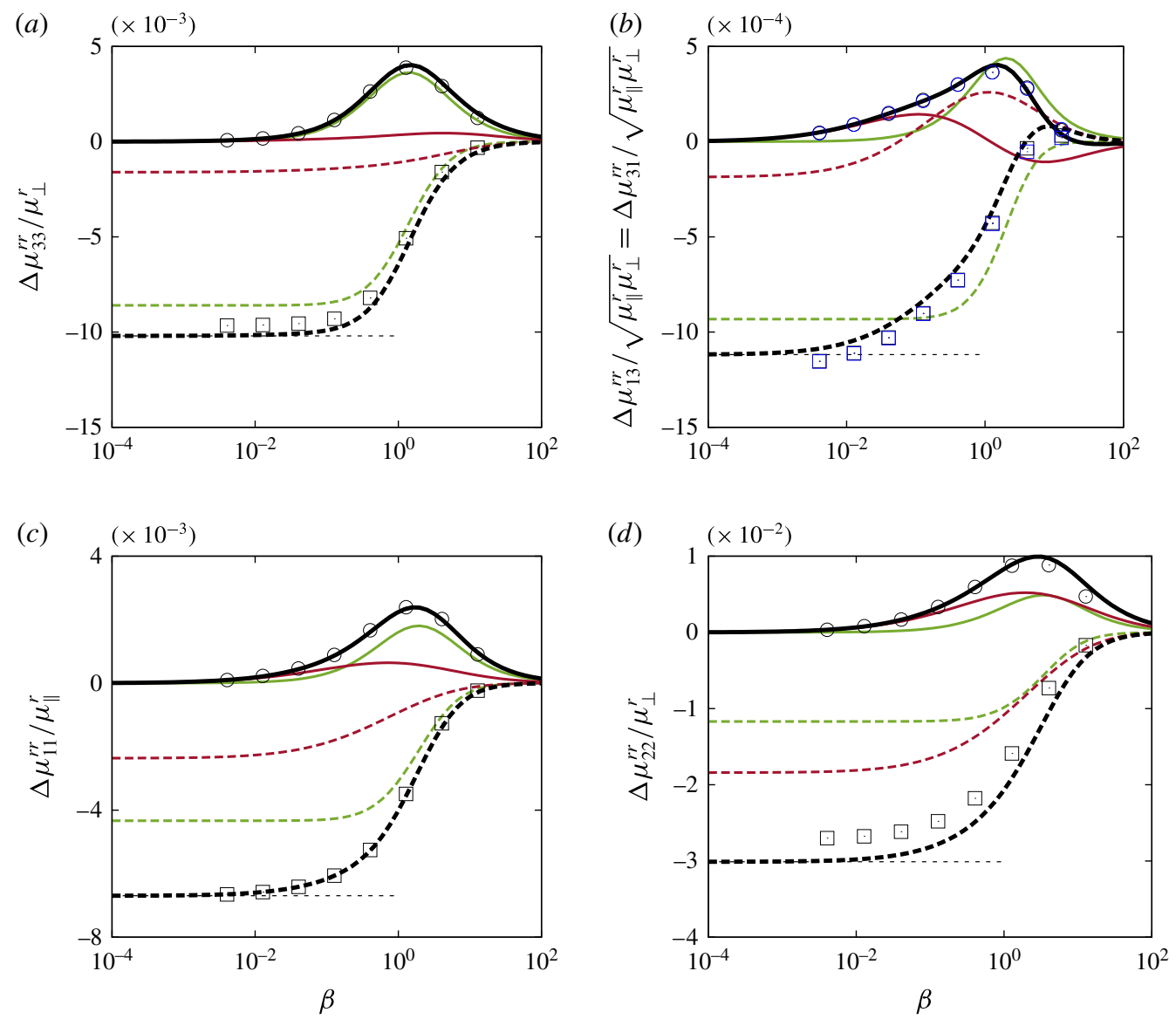

FIGURE 4. (Colour online) The scaled rotational mobility correction component versus the scaled frequency. Black and blue symbols refer to the 13 and 31 components, respectively, obtained from BIM simulations. The colour code is the same as in figure 2.

predictions and simulations. The coupling terms are generally very small compared to the relevant bulk quantities. This makes them somewhat more difficult to obtain precisely from the simulations which explains the small discrepancy notable in figure $3(a)$.

In figure 4 we present the corrections to the components of the rotational mobility tensor as calculated by (5.20)-(5.23) compared to the BIM simulations. We remark that the shearing contribution manifests itself in a more pronounced way for the rotational mobilities. Moreover, the correction to the rotational motion is less noticeable compared to the translational motion especially for the off-diagonal component. This observation can be explained by the fact that the rotational mobility corrections exhibit a faster decay with the distance from the membrane, scaling as $z_{0}^{-3}$ compared to $z_{0}^{-1}$ for translational motion. Again, a good agreement is obtained for the rotational mobility corrections between analytical predictions and numerical simulations. 


\section{Conclusions}

In this paper we have computed the leading-order translational, rotational and translation-rotation coupling hydrodynamic mobilities of an axisymmetric particle of arbitrary shape immersed in a Newtonian fluid in the vicinity of an elastic cell membrane. The resulting equations contain (i) the particle-independent multipole elements of the near-membrane Green's integral operator which have been calculated in analytical form in the present work and (ii) the mobility tensor of the particle in bulk. The mobility corrections are frequency-dependent complex quantities due to the memory induced by the membrane. They are expressed in terms of the particle orientation and two dimensionless parameters $\beta$ and $\beta_{B}$ that account for the shearing and bending-related contributions, respectively. In the zero-frequency limit, or equivalently for infinite elastic and bending moduli, we recover the mobilities near a hard no-slip wall. We apply our general formalism to a prolate spheroid and find very good agreement with numerical simulations performed for a truly extended spheroidal particle over the whole frequency spectrum.

\section{Acknowledgements}

A.D.-M.-I. and S.G. thank the Volkswagen Foundation for financial support and acknowledge the Gauss Center for Supercomputing e.V. for providing computing time on the GCS Supercomputer SuperMUC at Leibniz Supercomputing Center. This work has been supported by the Ministry of Science and Higher Education of Poland via the Mobility Plus Fellowship awarded to M.L. This article is based upon work from COST Action MP1305, supported by COST (European Cooperation in Science and Technology).

\section{Supplementary material}

Supplementary material is available at https://doi.org/10.1017/jfm.2016.739.

\section{Appendix A. The Green's function for an elastic membrane}

The Green's functions for an elastic membrane have been derived and discussed in detail in earlier papers (Daddi-Moussa-Ider et al. 2016a; Daddi-Moussa-Ider \& Gekle 2016). Here, we only sketch the derivation which starts with a two-dimensional Fourier transform of the Stokes equations and boundary conditions. It is convenient to introduce an orthogonal basis in the $x y$ plane, spanned by the unit vectors $\boldsymbol{e}_{l}=\boldsymbol{q} /|\boldsymbol{q}|$ and $\boldsymbol{e}_{t}=\boldsymbol{e}_{z} \times \boldsymbol{e}_{l}$, respectively parallel and perpendicular to the wave vector $\boldsymbol{q}$. This basis is rotated by the angle $\phi=\arctan \left(q_{y} / q_{x}\right)$ with respect to the laboratory frame.

After the pressure has been eliminated from the Fourier-transformed momentum equations, the following set of ordinary differential equations is obtained

$$
\begin{aligned}
q^{2} \tilde{v}_{t}-\tilde{v}_{t, z z} & =\frac{\tilde{F}_{t}}{\eta} \delta\left(z-z_{0}\right), \\
\tilde{v}_{z, z z z z}-2 q^{2} \tilde{v}_{z, z z}+q^{4} \tilde{v}_{z} & =\frac{q^{2} \tilde{F}_{z}}{\eta} \delta\left(z-z_{0}\right)+\frac{\mathrm{i} q \tilde{F}_{l}}{\eta} \delta^{\prime}\left(z-z_{0}\right), \\
\tilde{v}_{l} & =\frac{\mathrm{i} \tilde{v}_{z, z}}{q},
\end{aligned}
$$

where $\delta^{\prime}$ is the derivative of the Dirac delta function. After some algebra, it can be shown that the traction jump due to shearing as stated in (2.6) imposes at $z=0$ the 
following discontinuities

$$
\left[\tilde{v}_{t, z}\right]=-\left.\mathrm{i} B \alpha q^{2} \tilde{v}_{t}\right|_{z=0}, \quad\left[\tilde{v}_{z, z z}\right]=-\left.4 \mathrm{i} \alpha q^{2} \tilde{v}_{z, z}\right|_{z=0},
$$

where $\alpha:=\kappa_{S} / 3 B \eta \omega$ is a characteristic length for shear and area dilatation with $B:=$ $2 /(1+C)$. The normal traction jump as given by (2.7) leads to

$$
\left[\tilde{v}_{z, z z z}\right]=\left.4 \mathrm{i} \alpha_{B}^{3} q^{6} \tilde{v}_{z}\right|_{z=0},
$$

where $\alpha_{B}^{3}:=\kappa_{B} / 4 \eta \omega$, with $\alpha_{B}$ being a characteristic length for bending. The dimensionless numbers $\beta$ and $\beta_{B}$ stated in (2.9) are defined as $\beta:=2 z_{0} / \alpha$ and $\beta_{B}:=2 z_{0} / \alpha_{B}$.

The Green's tensor in this basis $\left\{\boldsymbol{e}_{l}, \boldsymbol{e}_{t}, \boldsymbol{e}_{z}\right\}$ has the form

$$
\tilde{\mathcal{G}}(q, z, \omega)=\left(\begin{array}{ccc}
\tilde{\mathcal{G}}_{l l} & 0 & \tilde{\mathcal{G}}_{l z} \\
0 & \tilde{\mathcal{G}}_{t t} & 0 \\
\tilde{\mathcal{G}}_{z l} & 0 & \tilde{\mathcal{G}}_{z z}
\end{array}\right) .
$$

The components of the Green's functions for $z \geqslant 0$ are expressed by

$$
\begin{aligned}
& \tilde{\mathcal{G}}_{z z}=\frac{1}{4 \eta q}\left(\left(1+q\left|z-z_{0}\right|\right) \mathrm{e}^{-q\left|z-z_{0}\right|}+\left(\frac{\mathrm{i} \alpha z z_{0} q^{3}}{1-\mathrm{i} \alpha q}+\frac{\mathrm{i} \alpha_{B}^{3} q^{3}(1+q z)\left(1+q z_{0}\right)}{1-\mathrm{i} \alpha_{B}^{3} q^{3}}\right) \mathrm{e}^{-q\left(z+z_{0}\right)}\right), \\
& \tilde{\mathcal{G}}_{l l}=\frac{1}{4 \eta q}\left(\left(1-q\left|z-z_{0}\right|\right) \mathrm{e}^{-q\left|z-z_{0}\right|}+\left(\frac{\mathrm{i} \alpha q\left(1-q z_{0}\right)(1-q z)}{1-\mathrm{i} \alpha q}+\frac{\mathrm{i} z z_{0} \alpha_{B}^{3} q^{5}}{1-\mathrm{i} \alpha_{B}^{3} q^{3}}\right) \mathrm{e}^{-q\left(z+z_{0}\right)}\right), \\
& \tilde{\mathcal{G}}_{t t}=\frac{1}{2 \eta q}\left(\mathrm{e}^{-q\left|z-z_{0}\right|}+\frac{\mathrm{i} B \alpha q}{2-\mathrm{i} B \alpha q} \mathrm{e}^{-q\left(z+z_{0}\right)}\right),
\end{aligned}
$$

with the off-diagonal components

$$
\begin{aligned}
& \tilde{\mathcal{G}}_{l z}=\frac{\mathrm{i}}{4 \eta q}\left(-q\left(z-z_{0}\right) \mathrm{e}^{-q\left|z-z_{0}\right|}+\left(\frac{\mathrm{i} \alpha z_{0} q^{2}(1-q z)}{1-\mathrm{i} \alpha q}-\frac{\mathrm{i} \alpha_{B}^{3} z q^{4}\left(1+q z_{0}\right)}{1-\mathrm{i} \alpha_{B}^{3} q^{3}}\right) \mathrm{e}^{-q\left(z+z_{0}\right)}\right), \\
& \tilde{\mathcal{G}}_{z l}=\frac{\mathrm{i}}{4 \eta q}\left(-q\left(z-z_{0}\right) \mathrm{e}^{-q\left|z-z_{0}\right|}+\left(-\frac{\mathrm{i} \alpha z q^{2}\left(1-q z_{0}\right)}{1-\mathrm{i} \alpha q}+\frac{\mathrm{i} \alpha_{B}^{3} q^{4} z_{0}(1+q z)}{1-\mathrm{i} \alpha_{B}^{3} q^{3}}\right) \mathrm{e}^{-q\left(z+z_{0}\right)}\right) .
\end{aligned}
$$

The terms which contain $\mathrm{e}^{-q\left|z-z_{0}\right|}$ are the Fourier-transformed elements of the Oseen tensor and do not depend on the elastic properties of the membrane. The remaining part comes from interactions with the interface. We now back transform (A 4) to the laboratory frame. Defining

$$
\tilde{\mathcal{G}}_{ \pm}(q, z, \omega):=\tilde{\mathcal{G}}_{t t}(q, z, \omega) \pm \tilde{\mathcal{G}}_{l l}(q, z, \omega),
$$

and performing the inverse spatial Fourier transform (Bracewell 1999), we find that the Green's functions for a point force acting at $\boldsymbol{r}_{0}=\left(0,0, z_{0}\right)$ can be presented in terms of the following convergent infinite integrals

$$
\mathcal{G}_{z z}(\boldsymbol{r}, \omega)=\frac{1}{2 \pi} \int_{0}^{\infty} \tilde{\mathcal{G}}_{z z}\left(q, z, z_{0}, \omega\right) J_{0}(\rho q) q \mathrm{~d} q
$$




$$
\begin{aligned}
& \mathcal{G}_{x x}(\boldsymbol{r}, \omega)=\frac{1}{4 \pi} \int_{0}^{\infty}\left(\tilde{\mathcal{G}}_{+}\left(q, z, z_{0}, \omega\right) J_{0}(\rho q)+\tilde{\mathcal{G}}_{-}\left(q, z, z_{0}, \omega\right) J_{2}(\rho q) \cos 2 \Theta\right) q \mathrm{~d} q, \\
& \mathcal{G}_{y y}(\boldsymbol{r}, \omega)=\frac{1}{4 \pi} \int_{0}^{\infty}\left(\tilde{\mathcal{G}}_{+}\left(q, z, z_{0}, \omega\right) J_{0}(\rho q)-\tilde{\mathcal{G}}_{-}\left(q, z, z_{0}, \omega\right) J_{2}(\rho q) \cos 2 \Theta\right) q \mathrm{~d} q, \\
& \mathcal{G}_{x y}(\boldsymbol{r}, \omega)=\frac{\sin 2 \Theta}{4 \pi} \int_{0}^{\infty} \tilde{\mathcal{G}}_{-}\left(q, z, z_{0}, \omega\right) J_{2}(\rho q) q \mathrm{~d} q, \\
& \mathcal{G}_{r z}(\boldsymbol{r}, \omega)=\frac{\mathrm{i}}{2 \pi} \int_{0}^{\infty} \tilde{\mathcal{G}}_{l z}\left(q, z, z_{0}, \omega\right) J_{1}(\rho q) q \mathrm{~d} q, \\
& \mathcal{G}_{z r}(\boldsymbol{r}, \omega)=\frac{\mathrm{i}}{2 \pi} \int_{0}^{\infty} \tilde{\mathcal{G}}_{z l}\left(q, z, z_{0}, \omega\right) J_{1}(\rho q) q \mathrm{~d} q,
\end{aligned}
$$

where $\rho:=\sqrt{x^{2}+y^{2}}$ is the radial distance from the origin, and $\Theta:=\arctan (y / x)$ is the angle formed by the radial and $x$ axis. Furthermore, $\mathcal{G}_{x z}=\mathcal{G}_{r z} \cos \Theta, \mathcal{G}_{y z}=\mathcal{G}_{r z} \sin \Theta$, $\mathcal{G}_{z x}=\mathcal{G}_{z r} \cos \Theta, \mathcal{G}_{z y}=\mathcal{G}_{z r} \sin \Theta$ and $\mathcal{G}_{y x}=\mathcal{G}_{x y}$. Here $J_{n}$ denotes the Bessel function (Abramowitz \& Stegun 1972) of the first kind of order $n$.

In the vanishing frequency limit, or equivalently for infinite membrane shearing and bending rigidities, the well-known Blake tensor (Blake 1971) is recovered for all the components of the Green's functions.

\section{Appendix B. Derivation of general mobility relations}

Here we sketch the manipulations that lead from the corrected friction tensor, given by (3.2) through (3.5), to the mobility correction in (3.7) through (3.10). We shall focus on the $t t$ part only, since the others follow analogously. Relation (2.5), rewritten as $\boldsymbol{\mu} \zeta=\mathbf{1}$, defines the relations between elements of the friction and mobility tensors of a particle close to a membrane. The membrane-corrected $t t$ friction tensor and the membrane-corrected $t t$ mobility are thus related by

$$
\begin{aligned}
& \boldsymbol{\mu}^{t t} \zeta^{t t}+\boldsymbol{\mu}^{t r} \zeta^{r t}=\mathbf{1} \\
& \boldsymbol{\mu}^{t t} \zeta^{t r}+\boldsymbol{\mu}^{t r} \zeta^{r r}=\mathbf{0}
\end{aligned}
$$

from which we have

$$
\mu^{t t}=\left[\zeta^{t t}-\zeta^{t r}\left(\zeta^{r r}\right)^{-1} \zeta^{r t}\right]^{-1}
$$

We know from (3.2) to (3.5) that the corrected friction has the following structure

$$
\begin{aligned}
& \zeta^{t t}=\zeta_{0}^{t t}+\Delta \zeta^{t t}, \\
& \zeta^{r t}=\zeta_{0}^{r r}+\Delta \zeta^{r r}, \\
& \zeta^{t r}=\Delta \zeta^{t r}, \\
& \zeta^{r t}=\Delta \zeta^{r t},
\end{aligned}
$$

with the known distance dependence of these elements. Moreover, for an axially symmetric particle, we have

$$
\zeta_{0}^{t t}=\left(\mu_{0}^{t t}\right)^{-1}, \quad \zeta_{0}^{r r}=\left(\mu_{0}^{r r}\right)^{-1}
$$


since the bulk friction and mobility tensors are diagonal. We now rewrite (B 3) as

$$
\boldsymbol{\mu}^{t t}=\boldsymbol{\mu}_{0}^{t t}\left[\mathbf{1}+\left(\Delta \zeta^{t t}\right) \boldsymbol{\mu}_{0}^{t t}-\left(\Delta \zeta^{t r}\right)\left(\zeta_{0}^{r r}+\Delta \zeta^{r r}\right)^{-1}\left(\Delta \zeta^{r t}\right) \boldsymbol{\mu}_{0}^{t t}\right]^{-1}
$$

and expand the expression $1 /(1+\delta)=1-\delta+\delta^{2}-\ldots$ around the bulk quantities. Restricting to quantities decaying slower than $z_{0}^{-3}$, we immediately find (3.7). An analogous procedure leads to the $t r, r t$ and $r r$ mobilities, where the elements of the bulk friction and mobility tensors combine to contribute only in the form of $\boldsymbol{\mu}_{0}^{d r}=\boldsymbol{\mu}_{0}^{r r} \zeta_{0}^{r d}$ and $\boldsymbol{\mu}_{0}^{r d}=-\boldsymbol{\zeta}_{0}^{d r} \boldsymbol{\mu}_{0}^{r r}$. The latter relations follow from the definitions (2.3) and (2.4).

\section{Appendix C. Expressions required for the spheroid mobilities}

The results for the correction are given in terms of the wall-particle distance $z_{0}$, its inclination angle $\theta$ and functions denoted by capital letters in (5.13) through (5.23) of the dimensionless shearing and bending parameters, $\beta$ and $\beta_{B}$. Below, we provide explicit expressions for these functions. They can conveniently be expressed in terms of higher-order exponential integrals (Abramowitz \& Stegun 1972). The contributions from the membrane shearing (index S) and bending (index B) are given separately. By summing up both, we arrive at the final expressions. Notably, in the limit of vanishing frequency, our results are in complete agreement with those given by Lisicki (2015), Lisicki et al. (2016).

\section{C.1. Translational mobility}

For the functions $P$ and $Q$, we find the shearing contribution as

$$
\begin{aligned}
& P_{S}(\beta)=-\frac{5}{4}+\frac{\beta^{2}}{8}-\frac{3 \mathrm{i} \beta}{8}+\frac{2 \mathrm{i} \beta}{B} \Gamma_{2}+\left(-\frac{\beta^{2}}{2}+\frac{\mathrm{i} \beta}{2}\left(1-\frac{\beta^{2}}{4}\right)\right) \mathrm{e}^{\mathrm{i} \beta} E_{1}(\mathrm{i} \beta), \\
& Q_{S}(\beta)=-\frac{3}{2} \mathrm{e}^{\mathrm{i} \beta} E_{4}(\mathrm{i} \beta),
\end{aligned}
$$

and the bending part

$$
\begin{aligned}
P_{B}\left(\beta_{B}\right)= & -\frac{1}{4}+\frac{\mathrm{i} \beta_{B}^{3}}{24}\left(\phi_{+}+\Gamma_{B}\right), \\
Q_{B}\left(\beta_{B}\right)= & -\frac{5}{2}+\mathrm{i} \beta_{B}\left(\left(\frac{\beta_{B}^{2}}{12}+\frac{\mathrm{i} \beta_{B}}{6}+\frac{1}{6}\right) \phi_{+}+\left(\frac{\beta_{B}^{2}}{12}-\frac{\mathrm{i} \beta_{B}}{3}-\frac{1}{3}\right) \mathrm{e}^{-\mathrm{i} \beta_{B}} E_{1}\left(-\mathrm{i} \beta_{B}\right)\right. \\
& \left.+\frac{\sqrt{3}}{6}\left(\beta_{B}+\mathrm{i}\right) \phi_{-}\right)
\end{aligned}
$$

with

$$
\begin{aligned}
\phi_{ \pm} & =\mathrm{e}^{-\mathrm{i} \overline{z_{B}}} E_{1}\left(-\mathrm{i} \overline{z_{B}}\right) \pm \mathrm{e}^{-\mathrm{i} z_{B}} E_{1}\left(-\mathrm{i} z_{B}\right), \\
\Gamma_{2} & =\mathrm{e}^{(2 \mathrm{i} \beta) / B} E_{1}\left(\frac{2 \mathrm{i} \beta}{B}\right), \\
\Gamma_{B} & =\mathrm{e}^{-\mathrm{i} \beta_{B}} E_{1}\left(-\mathrm{i} \beta_{B}\right),
\end{aligned}
$$

where $z_{B}:=\beta_{B} \mathrm{e}^{2 \mathrm{i} \pi / 3}$ and the bar denotes the complex conjugate. The function $E_{n}$ is the generalised exponential integral, $E_{n}(x)=\int_{1}^{\infty} t^{-n} \mathrm{e}^{-x t} \mathrm{~d} t$. 


\section{C.2. Translation-rotation coupling}

The translation-rotation coupling is determined by the functions $M$ and $N$, which we similarly decompose into two parts. Recalling that $B=2 /(1+C)$, the shearing part reads

$$
\begin{aligned}
& M_{S}(\beta)=\frac{3}{4}-\mathrm{i} \beta\left(\frac{1}{4}+\frac{1}{B}\right)+\frac{3 \beta^{2}}{8}+\frac{\mathrm{i} \beta^{3}}{8}-\frac{\beta^{2}}{2}\left(1+\mathrm{i} \beta-\frac{\beta^{2}}{4}\right) \Gamma_{1}-\frac{2 \beta^{2}}{B^{2}} \Gamma_{2}, \\
& N_{S}(\beta)=-\frac{3}{4}+\mathrm{i} \beta\left(\frac{1}{2}+\frac{2}{B}\right)-\frac{3 \beta^{2}}{8}+\frac{\mathrm{i} \beta^{3}}{8}+\beta^{2}\left(1+\frac{\mathrm{i} \beta}{4}+\frac{\beta^{2}}{8}\right) \Gamma_{1}+\frac{4 \beta^{2}}{B^{2}} \Gamma_{2}
\end{aligned}
$$

while the bending part is

$$
\begin{aligned}
& M_{B}\left(\beta_{B}\right)=\frac{3}{4}-\frac{\mathrm{i} \beta_{B}^{3}}{8}+\frac{\beta_{B}^{4}}{24} \Gamma_{B}+\frac{\beta_{B}^{3}}{24} \psi, \\
& N_{B}\left(\beta_{B}\right)=\frac{9}{4}-\frac{\mathrm{i} \beta_{B}^{3}}{8}-\frac{\beta_{B}^{3}}{4}\left(\mathrm{i}-\frac{\beta_{B}}{6}\right) \Gamma_{B}+\frac{\beta_{B}^{3}}{24} \psi-\frac{\mathrm{i} \beta_{B}^{3}}{4} \phi_{+},
\end{aligned}
$$

where we defined

$$
\begin{aligned}
\Gamma_{1} & =\mathrm{e}^{\mathrm{i} \beta} E_{1}(\mathrm{i} \beta), \\
\psi & =\overline{z_{B}} \mathrm{e}^{-\mathrm{i} \overline{z_{B}}} E_{1}\left(-\mathrm{i} \overline{z_{B}}\right)+z_{B} \mathrm{e}^{-\mathrm{i} z_{B}} E_{1}\left(-\mathrm{i} z_{B}\right) .
\end{aligned}
$$

\section{C.3. Rotational mobility}

The rotational mobility is described by a set of functions. The functions $A_{0}$ and $A_{2}$ defined for the component $\Delta \mu_{11}^{r r}$ in (5.20) are given by

$$
\begin{aligned}
& A_{0, S}(\beta)=-\frac{3}{2}+\frac{\mathrm{i} \beta}{2}\left(1+\frac{1}{B}\right)+\beta^{2}\left(\frac{1}{2}+\frac{1}{B^{2}}\right)-\frac{\mathrm{i} \beta^{3}}{2} \Gamma_{1}-\frac{2 \mathrm{i} \beta^{3}}{B^{3}} \Gamma_{2}, \\
& A_{2, S}(\beta)=\frac{1}{2}+\frac{\mathrm{i} \beta}{2}\left(\frac{1}{B}-1\right)+\beta^{2}\left(\frac{1}{B^{2}}-\frac{1}{2}\right)+\frac{\mathrm{i} \beta^{3}}{2} \Gamma_{1}-\frac{2 \mathrm{i} \beta^{3}}{B^{3}} \Gamma_{2}, \\
& A_{0, B}\left(\beta_{B}\right)=-A_{2, B}\left(\beta_{B}\right)=-1+\frac{\mathrm{i} \beta_{B}^{3}}{6}\left(\phi_{+}+\Gamma_{B}\right) .
\end{aligned}
$$

For the component $\Delta \mu_{13}^{r r}$, the function $D$ defined in (5.21) is given by

$$
\begin{aligned}
D_{S}(\beta)= & -\frac{1}{2}+\frac{\mathrm{i} \beta}{2}\left(1-\frac{1-\lambda}{B}\right)+\beta^{2}\left(\frac{1}{2}+\frac{\lambda}{4}-\frac{1-\lambda}{B^{2}}\right) \\
& +\mathrm{i} \beta^{3}\left(\frac{2(1-\lambda)}{B^{3}} \Gamma_{2}-\frac{\lambda+1}{2} \Gamma_{1}+\frac{\lambda}{4}\right)+\frac{\lambda \beta^{4}}{4} \Gamma_{1}, \\
D_{B}\left(\beta_{B}\right)= & -1+\frac{3 \lambda}{2}+\frac{\beta_{B}^{3}}{4}\left(-\mathrm{i} \lambda+\frac{2 \mathrm{i}}{3} \Gamma_{B}+\frac{2 \mathrm{i} \phi_{+}+\lambda \psi}{3}\right)+\frac{\lambda \beta_{B}^{4}}{12} \Gamma_{B} .
\end{aligned}
$$

Further, the shearing-related parts of $C_{0}, C_{2}$ and $C_{4}$ as defined for the correction $\Delta \mu_{22}^{r r}$ in (5.22) read

$$
C_{0, S}(\beta)=-\frac{3}{2}\left(1+\lambda^{2}\right)+\left(\frac{\mathrm{i} \lambda^{2} \beta^{2}}{8}-\frac{\lambda(1-\lambda) \beta}{2}-\frac{\mathrm{i}(1-\lambda)^{2}}{2}\right) \beta^{3} \Gamma_{1}-\frac{2 \mathrm{i}}{B^{3}}(1-\lambda)^{2} \beta^{3} \Gamma_{2}
$$




$$
\begin{aligned}
& +\frac{\mathrm{i} \beta}{4}\left(\lambda^{2}+2+\frac{2(1-\lambda)^{2}}{B}\right)+\frac{\beta^{2}}{4}\left(\lambda^{2}-2 \lambda+2+\frac{4(1-\lambda)^{2}}{B^{2}}\right) \\
& -\mathrm{i} \lambda \beta^{3}\left(\frac{1}{2}-\frac{3 \lambda}{8}\right)-\frac{\lambda^{2} \beta^{4}}{8}, \\
C_{2, S}(\beta)= & \frac{3 \lambda}{4}-\beta^{3}(2+\mathrm{i} \beta)\left(\mathrm{i}-\frac{3}{32} \lambda(\beta-2 i)\right) \Gamma_{1}-\frac{2 \mathrm{i}}{B^{3}}(4-3 \lambda) \beta^{3} \Gamma_{2} \\
& +\frac{\mathrm{i} \beta}{2}\left(\frac{3 \lambda}{8}+\frac{4-3 \lambda}{B}\right) \\
& +\beta^{2}\left(\frac{3 \lambda}{16}+1+\frac{4-3 \lambda}{B^{2}}\right)+\mathrm{i} \beta^{3}\left(\frac{9 \lambda}{32}+1\right)-\frac{3 \lambda}{32} \beta^{4}, \\
C_{4, S}(\beta)= & -\frac{3}{4}+3 \mathrm{i} \beta\left(-\frac{1}{16}+\frac{1}{2 B}\right)+3 \beta^{2}\left(-\frac{1}{16}+\frac{1}{B^{2}}\right)+\mathrm{i} \beta^{3}\left(-\frac{9}{32}-\frac{6}{B^{3}} \Gamma_{2}\right) \\
& +\frac{3}{8} \beta^{3}\left(\mathrm{i}-\beta-\frac{\mathrm{i}}{4} \beta^{2}\right) \Gamma_{1}+\frac{3}{32} \beta^{4},
\end{aligned}
$$

and the bending-related parts read

$$
\begin{aligned}
C_{0, B}\left(\beta_{B}\right)= & -1-3 \lambda(1+\lambda)+\frac{\mathrm{i} \beta_{B}^{3}}{8} \lambda(\lambda+4)-\frac{\beta_{B}^{3}}{24}\left(\mathrm{i} \lambda^{2} \beta_{B}^{2}+4 \lambda \beta_{B}-4 \mathrm{i}\right) \Gamma_{B} \\
& +\frac{\beta_{B}^{3}}{6}\left(\mathrm{i} \phi_{+}-\lambda \psi+\frac{\mathrm{i} \lambda^{2}}{4} \beta_{B}\left(\psi+\beta_{B} \phi_{+}\right)\right), \\
C_{2, B}\left(\beta_{B}\right)= & 6-\frac{9}{4} \lambda-\mathrm{i} \beta_{B}^{3}\left(1-\frac{3 \lambda}{32}\right)+\left(\frac{1}{3}-\frac{\mathrm{i} \lambda \beta_{B}}{32}\right) \beta_{B}^{4} \Gamma_{B}+\frac{\beta_{B}^{3}}{3} \psi \\
& +\frac{\mathrm{i} \lambda \beta_{B}^{4}}{32}\left(\psi+\beta_{B} \phi_{+}\right), \\
C_{4, B}\left(\beta_{B}\right)= & \frac{9}{4}-\frac{3 \mathrm{i} \beta_{B}^{3}}{32}+\frac{\mathrm{i} \beta_{B}^{5}}{32} \Gamma_{B}-\frac{\mathrm{i} \beta_{B}^{4}}{32}\left(\psi+\beta_{B} \phi_{+}\right),
\end{aligned}
$$

Finally, the functions $H_{0}$ and $H_{2}$ defined for the component $\Delta \mu_{33}^{r r}$ in (5.23) read

$$
\begin{aligned}
H_{0, S}(\beta)= & -1-\frac{3}{4} \lambda^{2}+\mathrm{i} \beta\left(\frac{\lambda^{2}+2}{2 B}+\frac{\lambda^{2}}{16}\right)+\beta^{2}\left(\frac{2+\lambda^{2}}{B^{2}}+\frac{\lambda^{2}}{16}\right) \\
& +\frac{\lambda^{2} \beta^{3}}{32}\left(\mathrm{i} \beta^{2}-4 \mathrm{i}+4 \beta\right) \Gamma_{1} \\
& +\beta^{3}\left(\frac{3 \mathrm{i} \lambda^{2}}{32}-\frac{2 \mathrm{i}}{B^{3}}\left(\lambda^{2}+2\right) \Gamma_{2}\right)-\frac{\lambda^{2} \beta^{4}}{32}, \\
H_{2, S}(\beta)= & -\frac{1}{2}-\frac{3}{4} \lambda^{2}+\beta\left(\frac{3 \mathrm{i}}{16} \lambda^{2}+\frac{\mathrm{i} \lambda}{B}+\frac{\mathrm{i}}{2}-\frac{\mathrm{i}}{2 B}\right)+\beta^{2}\left(\frac{3}{16} \lambda^{2}+\frac{\lambda+1}{2}+\frac{2 \lambda}{B^{2}}-\frac{1}{B^{2}}\right) \\
& +\frac{\beta^{3}}{32}\left(9 \mathrm{i} \lambda^{2}+16 \mathrm{i} \lambda+\frac{64 \mathrm{i}(1-2 \lambda) \Gamma_{2}}{B^{3}}+(4+6 \lambda+3 \mathrm{i} \lambda \beta)(\lambda \beta-2 \mathrm{i} \lambda-4 \mathrm{i}) \Gamma_{1}\right) \\
& -\frac{3 \lambda^{2} \beta^{4}}{32},
\end{aligned}
$$


and

$$
\begin{aligned}
H_{0, B}\left(\beta_{B}\right)= & \lambda^{2}\left(-\frac{3}{4}+\frac{\mathrm{i} \beta_{B}^{3}}{32}-\frac{\mathrm{i} \beta_{B}^{5}}{96} \Gamma_{B}+\frac{\mathrm{i} \beta_{B}^{4}}{96}\left(\psi+\beta_{B} \phi_{+}\right)\right), \\
H_{2, B}\left(\beta_{B}\right)= & -1+3 \lambda-\frac{9}{4} \lambda^{2}+\beta_{B}^{3}\left(\mathrm{i} \lambda\left(\frac{3}{32} \lambda-\frac{1}{2}\right)+\frac{1}{6}\left(\mathrm{i}+\lambda \beta_{B}-\frac{3 \mathrm{i} \lambda^{2} \beta_{B}^{2}}{16}\right) \Gamma_{B}\right) \\
& +\frac{\mathrm{i} \lambda^{2} \beta_{B}^{4}}{32}\left(\psi+\beta_{B} \phi_{+}\right)+\frac{\beta_{B}^{3}}{6}\left(\lambda \psi+\mathrm{i} \phi_{+}\right) .
\end{aligned}
$$

\section{REFERENCES}

Abramowitz, M. \& Stegun, I. A. 1972 Handbook of Mathematical Functions, vol. 1. Dover. Agudo-Canalejo, J. \& Lipowsky, R. 2015 Critical particle sizes for the engulfment of nanoparticles by membranes and vesicles with bilayer asymmetry. ACS Nano 9 (4), 3704-3720.

Al-Obaidi, H. \& Florence, A. T. 2015 Nanoparticle delivery and particle diffusion in confined and complex environments. J. Drug Deliv. Sci. Technol. 30, 266-277.

BAtChELOR, G. K. 1970 Slender-body theory for particles of arbitrary cross-section in Stokes flow. J. Fluid Mech. 44 (03), 419-440.

Bhaduri, B., Neild, A. \& NG, T. W. 2008 Directional Brownian diffusion dynamics with variable magnitudes. Appl. Phys. Lett. 92 (8), 084105.

Bickel, T. 2006 Brownian motion near a liquid-like membrane. Eur. Phys. J. E 20, 379-385.

BICKel, T. 2007 Hindered mobility of a particle near a soft interface. Phys. Rev. E 75, 041403.

BICKEL, T. 2014 Probing nanoscale deformations of a fluctuating interface. Europhys. Lett. 106 (1), 16004.

Blake, J. R. 1971 A note on the image system for a Stokeslet in a no-slip boundary. Math. Proc. Camb. Phil. Soc. 70 (02), 303-310.

Blake, J. R. \& Fulford, G. R. 1981 Force distribution on a slender body close to an interface. Bull. Austral. Math. Soc. 24 (01), 27-36.

BŁaWZdZIEWicz, J., EKIEL-JeŻewsKa, M. L. \& WAJnRyb, E. 2010 Motion of a spherical particle near a planar fluid-fluid interface: the effect of surface incompressibility. J. Chem. Phys. 133 (11), 114702.

Bohtwright, T., Dennin, M., Shlomovitz, R., Evans, A. A. \& Levine, A. J. 2014 Probing interfacial dynamics and mechanics using submerged particle microrheology. II. Experiment. Phys. Fluids 26 (7), 071904.

BraceWell, R. 1999 The Fourier Transform and its Applications. McGraw-Hill.

BRENNER, H. 1961 The slow motion of a sphere through a viscous fluid towards a plane surface. Chem. Engng Sci. 16, 242-251.

Cervantes-Martínez, A. E., Ramírez-Saito, A., Armenta-Calderón, R., Ojeda-López, M. A. \& Arauz-Lara, J. L. 2011 Colloidal diffusion inside a spherical cell. Phys. Rev. E 83 (3), 030402-4.

Cheong, F. C. \& Grier, D. G. 2010 Rotational and translational diffusion of copper oxide nanorods measured with holographic video microscopy. Opt. Express 18 (7), 6555-6562.

Chwang, A. T. \& WU, T. Y.-T. 1975 Hydromechanics of low-Reynolds-number flow. Part 2. Singularity method for Stokes flows. J. Fluid Mech. 67 (04), 787-815.

Cichocki, B. \& Jones, R. B. 1998 Image representation of a spherical particle near a hard wall. Physica A 258 (3), 273-302.

Cichocki, B., Jones, R. B., Kutteh, R. \& Wajnryb, E. 2000 Friction and mobility for colloidal spheres in Stokes flow near a boundary: The multipole method and applications. J. Chem. Phys. 112 (5), 2548-2561.

Colin, R., Yan, M., Chevry, L., Berret, J.-F. \& Abou, B. 2012 3d rotational diffusion of micrometric wires using 2d video microscopy. Europhys. Lett. 97 (3), 30008. 
Conn, A. R., Gould, N. I. M. \& Toint, Ph. L. 2000 Trust Region Methods, vol. 1. SIAM.

DAdDi-Moussa-Ider, A. \& GeKLE, S. 2016 Hydrodynamic interaction between particles near elastic interfaces. J. Chem. Phys. 145 (1), 014905.

Daddi-Moussa-Ider, A., Guckenberger, A. \& GeKLe, S. $2016 a$ Long-lived anomalous thermal diffusion induced by elastic cell membranes on nearby particles. Phys. Rev. E 93, 012612.

Daddi-Moussa-Ider, A., Guckenberger, A. \& Gekle, S. $2016 b$ Particle mobility between two planar elastic membranes: Brownian motion and membrane deformation. Phys. Fluids 28 (7), 071903.

De Corato, M., Greco, F., Davino, G. \& Maffettone, P. L. 2015 Hydrodynamics and Brownian motions of a spheroid near a rigid wall. J. Chem. Phys. 142 (19), 194901.

De Mestre, N. J. \& Russel, W. B. 1975 Low-Reynolds-number translation of a slender cylinder near a plane wall. J. Engng Maths 9 (2), 81-91.

Dettmer, S. L., Pagliara, S., Misiunas, K. \& Keyser, U. F. 2014 Anisotropic diffusion of spherical particles in closely confining microchannels. Phys. Rev. E 89 (6), 062305.

Doherty, G. J. \& McMahon, H. T. 2009 Mechanisms of endocytosis. Annu. Rev. Biochem. 78 (1), 857-902.

Dufresne, E. R., Altman, D. \& Grier, D. G. 2001 Brownian dynamics of a sphere between parallel walls. Europhys. Lett. 53 (2), 264-270.

Duggal, R. T \& PAsquali, M. 2006 Dynamics of individual single-walled carbon nanotubes in water by real-time visualization. Phys. Rev. Lett. 96 (24), 246104.

Eral, H. B., OH, J. M., van den Ende, D., Mugele, F. \& Duits, M. H. G. 2010 Anisotropic and hindered diffusion of colloidal particles in a closed cylinder. Langmuir 26 (22), 16722-16729.

Faucheux, L. P. \& Libchaber, A. J. 1994 Confined Brownian motion. Phys. Rev. E 49, 5158-5163.

FELDERHOF, B. U. $2006 a$ Effect of surface elasticity on the motion of a droplet in a viscous fluid. J. Chem. Phys. 125 (12), 124904.

FELDERHOF, B. U. $2006 b$ Effect of surface tension and surface elasticity of a fluid-fluid interface on the motion of a particle immersed near the interface. J. Chem. Phys. 125 (14), 144718.

FELDERHOF, B. U. 2012 Hydrodynamic force on a particle oscillating in a viscous fluid near a wall with dynamic partial-slip boundary condition. Phys. Rev. E 85, 046303.

FELDERHOF, B. U. 2013 Velocity relaxation of an ellipsoid immersed in a viscous incompressible fluid. Phys. Fluids 25 (1), 013101.

Franosch, T. \& Jeney, S. 2009 Persistent correlation of constrained colloidal motion. Phys. Rev. E 79 (3), 031402.

Freund, J. B. 2014 Numerical simulation of flowing blood cells. Annu. Rev. Fluid Mech. 46 (1), 67-95.

Goldman, A. J., Cox, R. G. \& Brenner, H. 1967a Slow viscous motion of a sphere parallel to a plane wall- I Motion through a quiescent fluid. Chem. Engng Sci. 22, 637-651.

Goldman, A. J., Cox, R. G. \& Brenner, H. $1967 b$ Slow viscous motion of a sphere parallel to a plane wall-II Couette flow. Chem. Engng Sci. 22, 653-660.

Guckenberger, A., Schraml, M. P., Chen, P. G., Leonetti, M. \& Gekle, S. 2016 On the bending algorithms for soft objects in flows. Comput. Phys. Commun. 207, 1-23.

Han, Y., Alsayed, A., Nobili, M. \& Yodh, A. G. 2009 Quasi-two-dimensional diffusion of single ellipsoids: Aspect ratio and confinement effects. Phys. Rev. E 80, 011403.

Han, Y., Alsayed, A. M., Nobili, M., Zhang, J., Lubensky, T. C. \& Yodh, A. G. 2006 Brownian motion of an ellipsoid. Science 314 (5799), 626-630.

Happel, J. \& Brenner, H. 2012 Low Reynolds Number Hydrodynamics: With Special Applications to Particulate Media, vol. 1. Springer Science \& Business Media.

Helfrich, W. 1973 Elastic properties of lipid bilayers - theory and possible experiments. Z. Naturf. C. $28,693$.

Holmqvist, P., Dhont, J. K. G. \& LAnG, P. R. 2007 Colloidal dynamics near a wall studied by evanescent wave light scattering: experimental and theoretical improvements and methodological limitations. J. Chem. Phys. 126 (4), 044707. 
Hsu, R. \& Ganatos, P. 1989 The motion of a rigid body in viscous fluid bounded by a plane wall. J. Fluid Mech. 207, 29-72.

Irmscher, M., De Jong, A. M., Kress, H. \& Prins, M. W. J. 2012 Probing the cell membrane by magnetic particle actuation and euler angle tracking. Biophys. J. 102 (3), 698-708.

Jünger, F., Kohler, F., Meinel, A., Meyer, T., Nitschke, R., Erhard, B. \& Rohrbach, A. 2015 Measuring local viscosities near plasma membranes of living cells with photonic force microscopy. Biophys. J. 109 (5), 869-882.

Kim, S. \& KARRILA, S. J. 2013 Microhydrodynamics: Principles and Selected Applications. Courier Corporation.

Kress, H., Stelzer, E. H. K., Griffiths, G. \& Rohrbach, A. 2005 Control of relative radiation pressure in optical traps: application to phagocytic membrane binding studies. Phys. Rev. E 71 (6), 061927.

Lauga, E. \& Squires, T. M. 2005 Brownian motion near a partial-slip boundary: A local probe of the no-slip condition. Phys. Fluids 17 (10), 103102.

Lee, S. H., Chadwick, R. S. \& Leal, L. G. 1979 Motion of a sphere in the presence of a plane interface. part 1. an approximate solution by generalization of the method of lorentz. J. Fluid Mech. 93, 705-726.

LI, G. \& TANG, J. X. 2004 Diffusion of actin filaments within a thin layer between two walls. Phys. Rev. E 69 (6), 061921.

LIN, B., YU, J. \& RICE, S. A. 2000 Direct measurements of constrained Brownian motion of an isolated sphere between two walls. Phys. Rev. E 62, 3909-3919.

LISICKI, M. 2015 Evanescent wave dynamic light scattering by optically anisotropic brownian particles. $\mathrm{PhD}$ thesis, University of Warsaw.

Lisicki, M., Cichocki, B., Dhont, J. K. G. \& LAng, P. R. 2012 One-particle correlation function in evanescent wave dynamic light scattering. J. Chem. Phys. 136 (20), 204704.

Lisicki, M., Cichocki, B., Rogers, S. A., Dhont, J. K. G. \& LANG, P. R. 2014 Translational and rotational near-wall diffusion of spherical colloids studied by evanescent wave scattering. Soft Matt. 10 (24), 4312-4323.

Lisicki, M., Cichocki, B. \& WAJNRYB, E. 2016 Near-wall diffusion tensor of an axisymmetric colloidal particle. J. Chem. Phys. 145, 034904.

Liu, J., Wei, T., Zhao, J., Huang, Y., Deng, H., Kumar, A., Wang, C., Liang, Z., Ma, X. \& LIANG, X.-J. 2016 Multifunctional aptamer-based nanoparticles for targeted drug delivery to circumvent cancer resistance. Biomaterials 91, 44-56.

LORENTZ, H. A. 1907 Ein allgemeiner satz, die bewegung einer reibenden flüssigkeit betreffend, nebst einigen anwendungen desselben. Abh. Theor. Phys. 1, 23.

Meinel, A., Tränkle, B., Römer, W. \& Rohrbach, A. 2014 Induced phagocytic particle uptake into a giant unilamellar vesicle. Soft Matt. 10 (20), 3667-3678.

Michailidou, V. N., Petekidis, G., Swan, J. W. \& Brady, J. F. 2009 Dynamics of concentrated hard-sphere colloids near a wall. Phys. Rev. Lett. 102, 068302.

Michailidou, V. N., Swan, J. W., Brady, J. F. \& Petekidis, G. 2013 Anisotropic diffusion of concentrated hard-sphere colloids near a hard wall studied by evanescent wave dynamic light scattering. J. Chem. Phys. 139 (16), 164905.

Mitchell, W. H. \& Spagnolie, S. E. 2015 Sedimentation of spheroidal bodies near walls in viscous fluids: glancing, reversing, tumbling, and sliding. J. Fluid Mech. 772, 600.

MukniJA, D. \& Solomon, M. J. 2007 Translational and rotational dynamics of colloidal rods by direct visualization with confocal microscopy. J. Colloid Interface Sci. 314 (1), 98-106.

NaAhidi, S., Jafari, M., Edalat, F., Raymond, K., Khademhosseini, A. \& Chen, P. 2013 Biocompatibility of engineered nanoparticles for drug delivery. J. Control. Release 166 (2), 182-194.

Neild, A., Padding, J. T., Yu, L., Bhaduri, B., Briels, W. J. \& NG, T. W. 2010 Translational and rotational coupling in Brownian rods near a solid surface. Phys. Rev. E 82 (4), 041126.

PADding, J. T. \& BRIELS, W. J. 2010 Translational and rotational friction on a colloidal rod near a wall. J. Chem. Phys. 132, 054511. 
PERrin, F. 1934 Mouvement brownien d'un ellipsoide - I. Dispersion diélectrique pour des molécules ellipsoidales. J. Phys. Radium 5, 497-511.

Perrin, F. 1936 Mouvement brownien d'un ellipsoide - II. Rotation libre et dépolarisation des fluorescences. translation et diffusion de molécules ellipsoidales. J. Phys. Radium 7, 1-11.

Power, H. \& Miranda, G. 1987 Second kind integral equation formulation of Stokes' flows past a particle of arbitrary shape. SIAM J. Appl. Maths 47 (4), 689-698.

Pozrikidis, C. 2001 Interfacial dynamics for Stokes flow. J. Comput. Phys. 169, 250.

Rogers, S. A., Lisicki, M., Cichocki, B., Dhont, J. K. G. \& LAng, P. R. 2012 Rotational diffusion of spherical colloids close to a wall. Phys. Rev. Lett. 109 (9), 098305.

Saintyves, B., Jules, T., Salez, T. \& Mahadevan, L. 2016 Self-sustained lift and low friction via soft lubrication. Proc. Natl Acad. Sci. USA 113 (21), 5847-5849.

SAlez, T. \& MAhadevan, L. 2015 Elastohydrodynamics of a sliding, spinning and sedimenting cylinder near a soft wall. J. Fluid Mech. 779, 181-196.

SCHÄFFER, E., NøRRELYKKe, S. F. \& HowARD, J. 2007 Surface forces and drag coefficients of microspheres near a plane surface measured with optical tweezers. Langmuir 23 (7), $3654-3665$.

SCHIBY, D. \& GALLILY, I. 1980 On the orderly nature of the motion of nonspherical aerosol particles. III. The effect of the particle-wall fluid-dynamic interaction. J. Colloid Interface Sci. 77 (2), $328-352$.

Shlomovitz, R., Evans, A., Boatwright, T., Dennin, M. \& Levine, A. 2013 Measurement of monolayer viscosity using noncontact microrheology. Phys. Rev. Lett. 110 (13), 137802.

Shlomovitz, R., Evans, A. A., Boatwright, T., Dennin, M. \& Levine, A. J. 2014 Probing interfacial dynamics and mechanics using submerged particle microrheology. I. Theory. Phys. Fluids 26 (7), 071903.

Skalak, R., Tozeren, A., Zarda, R. P. \& Chien, S. 1973 Strain energy function of red blood cell membranes. Biophys. J. 13 (3), 245-264.

SWAN, J. W. \& BRADY, J. F. 2007 Simulation of hydrodynamically interacting particles near a no-slip boundary. Phys. Fluids 19 (11), 113306.

Tränkle, B., RUh, D. \& Rohrbach, A. 2016 Interaction dynamics of two diffusing particles: contact times and influence of nearby surfaces. Soft Matt. 12 (10), 2729-2736.

Waigh, T. A. 2016 Advances in the microrheology of complex fluids. Rep. Prog. Phys. 79 (7), 074601 .

Wang, G. M., Prabhakar, R. \& Sevick, E. M. 2009 Hydrodynamic mobility of an optically trapped colloidal particle near fluid-fluid interfaces. Phys. Rev. Lett. 103, 248303.

WANG, W. \& HuAng, P. 2014 Anisotropic mobility of particles near the interface of two immiscible liquids. Phys. Fluids 26 (9), 092003.

Zhao, H. \& Shaqfeh, E. S. G. 2011 Shear-induced platelet margination in a microchannel. Phys. Rev. E 83, 061924.

Zhao, H., Shaqfeh, E. S. G. \& Narsimhan, V. 2012 Shear-induced particle migration and margination in a cellular suspension. Phys. Fluids 24 (1), 011902.

ZHENG, Z. \& HAN, Y. 2010 Self-diffusion in two-dimensional hard ellipsoid suspensions. J. Chem. Phys. 133 (12), 124509.

ZHU, L. 2014 Simulation of individual cells in flow. PhD thesis, KTH Royal Institute of Technology in Stockholm. 